OPEN ACCESS

Edited by:

Ana Ribeiro,

University Porto, Portugal

Reviewed by:

Marta Fadda,

University of Italian Switzerland,

Switzerland

Pablo Perel,

University of London, United Kingdom

Louise Hartley,

RTI Health Solutions, United Kingdom

*Correspondence:

Rawlance Ndejjo

rndejjo@musph.ac.ug

${ }^{+}$These authors have contributed

equally to this work

Received: 06 February 2021 Accepted: 07 May 2021

Published: 02 June 2021

Citation:

Ndejjo R, Hassen HY, Wanyenze RK,

Musoke D, Nuwaha F, Abrams S, Bastiaens $H$ and Musinguzi G (2021)

Community-Based Interventions for Cardiovascular Disease Prevention in Low-and Middle-Income Countries: A

Systematic Review.

Public Health Rev 42:1604018. doi: 10.3389/phrs.2021.1604018

\section{Community-Based Interventions for Cardiovascular Disease Prevention in Low-and Middle-Income Countries: A Systematic Review}

\author{
Rawlance Ndejjo ${ }^{1,2 *}$, Hamid Yimam Hassen ${ }^{2}$, Rhoda K. Wanyenze ${ }^{1}$, David Musoke ${ }^{1}$, \\ Fred Nuwaha ${ }^{1}$, Steven Abrams ${ }^{2,3}$, Hilde Bastiaens ${ }^{2 \dagger}$ and Geofrey Musinguzi ${ }^{1,2 \dagger}$ \\ ${ }^{1}$ Department of Disease Control and Environmental Health, School of Public Health, College of Health Sciences, Makerere \\ University, Kampala, Uganda, ${ }^{2}$ Department Family Medicine and Population Health, Faculty of Medicine and Health Sciences, \\ University of Antwerp, Antwerp, Belgium, ${ }^{3}$ Data Science Institute, Interuniversity Institute for Biostatistics and Statistical \\ Bioinformatics (I-BioStat), UHasselt, Belgium
}

Objectives: To synthesize evidence on the effectiveness of community-based interventions for cardiovascular disease (CVD) prevention in low- and middle-income countries (LMICs) to inform design of effective strategies for CVD prevention.

Methods: We searched MEDLINE, EMBASE, CINAHL, Cochrane register of controlled studies and PSYCINFO databases for studies published between January 2000 and June 2019. Other studies were identified from gray literature sources and review of reference lists of included studies. The primary outcomes for the review were those aimed at primary prevention of CVD targeting physical activity, diet, smoking and alcohol consumption.

Results: Database searches yielded 15,885 articles and 94 articles were identified through snowball searching. After screening, the articles from LMICs were 32 emanating from 27 studies: 9 cluster randomized trials, eight randomized controlled trials and 10 controlled before and after studies. Community-based interventions successfully improved population knowledge on CVD and risk factors and influenced physical activity and dietary practices. Evidence of interventions on smoking cessation and reduced alcohol consumption was inconsistent.

Conclusion: This evidence should inform policy makers in decision-making and prioritizing evidence-based interventions.

Keywords: smoking, cardiovascular disease, knowledge, physical activity, alcohol, community-based, diet, effectiveness

Abbreviations: CVD, Cardiovascular disease; LMICs, Low- and middle-income countries; NCDs, Non-communicable diseases; PRISMA, Preferred Reporting Items for Systematic Review and Meta-Analyses; RoB, Risk of Bias; ROBINS-I, Risk of Bias in Non-randomized Studies of Interventions; SPICES, Scaling up Packages of Interventions for Cardiovascular disease prevention in selected sites in Europe and sub-Saharan Africa. 


\section{INTRODUCTION}

Cardiovascular disease (CVD) continues to disproportionately cause morbidity and mortality in low- and middle-income countries (LMICs). Of the 17.9 million CVD related deaths reported worldwide in 2016, 75\% occurred in LMICs [1]. In many LMICs, epidemiological transition, industrialization, infectious diseases burden and globalization have influenced changes in lifestyle observed through changes in physical activity, diet, alcohol and smoking behavior among others [2]. These lifestyle changes have contributed to the upsurge in CVD metabolic risk factors such as obesity, hypertension and diabetes [2]. It is estimated that over half of the 671 million obese population in the world live in 10 countries, eight of which are LMICs [3]. Moreover, the number of people living with diabetes in LMICs is estimated to rise to 228 million by 2030 from 84 million in 1995 [4]. A 2015 systematic review reported a pooled prevalence of hypertension in LMICs of 32.3\% (95\% CI: 29.4-35.3) [5] while a prevalence of $57.0 \%$ (95\% CI 52-61\%) was reported in another review among the African population aged 50 years and above [6].

Targeting lifestyle factors such as physical inactivity, poor diets, smoking and alcohol intake, and metabolic risk factors including dyslipidemia, hypertension and diabetes can reduce the overall burden of CVD [7]. Community-based interventions target change among individuals, groups, and organizations to avoid development of CVD risk factors or control them and often incorporate strategies to create policy and environmental changes $[8,9]$. Through community-based interventions, reduction in CVD burden and risk can be achieved in entire communities impacting population level knowledge and perceptions and risk reduction practices $[10,11]$. Population level public health measures are also likely to be more cost effective [12] than treatment oriented programmes for which most LMICs lack capacity to implement on a large scale [13].

Although community-based interventions aimed at CVD prevention have been implemented in LMICs, gaps remain regarding their effectiveness in these settings. Previous reviews on community based interventions have not been specifically directed to LMICs [14, 15], evaluated only a few of the interventions or outcomes [16-18], or do not include recent evidence [19]. This systematic review was aimed at providing upto-date and comprehensive evidence on the effectiveness of community-based interventions for CVD prevention to support the design of effective strategies for CVD prevention. This review therefore answers two key research questions considering LMICs:

1) What community-based interventions and strategies have been implemented for CVD prevention in LMICs?

2) What is the effectiveness of community-based interventions for CVD prevention in LMICs?

\section{METHODS}

This study was conducted and reported in accordance with the Preferred Reporting Items for Systematic reviews and Meta-
Analysis Protocols (PRISMA-P) 2015 statement (S1 Checklist) [20]. The study protocol was registered in the Prospero International prospective register of systematic reviews (Registration Number: CRD42019119885).

\section{Eligibility Criteria}

This review included studies conducted between January 2000 and June 2019 to obtain recent relevant evidence on communitybased interventions for cardiovascular disease prevention to inform policy and practice applicable in the current social dynamics. Studies were included if they met the criteria below:

- Study population: Studies conducted among adults aged 18 years and above.

- Intervention: Studies that reported interventions carried out within the community for either primordial or primary prevention of CVD aimed to improve cardiovascular risk knowledge and healthy lifestyle such as physical activity, healthy dietary habit, cessation of smoking and alcohol consumption. These interventions include health education and promotion, community mobilization, lifestyle counseling and coaching, screening, and treatment. The studies ought to have been implemented in a community setting including households, workplaces, schools, sport centers, pharmacies, primary health care units, community health worker posts among others but not secondary care health facilities. Interventions that started at health facilities and later linked to the community were included.

- Comparator: studies where intervention was compared with another intervention, usual care or nothing.

- Outcomes: The primary outcomes were changes in knowledge regarding CVD, physical activity, diet, smoking, and alcohol consumption. Among studies that had at least one primary outcome, secondary outcomes of body weight, systolic and diastolic blood pressure, blood glucose and lipid levels were reported. Studies among patients with CVD conditions and those whose aim was not to prevent CVD or their risk factors were excluded.

- Study designs: Individual level or cluster randomized controlled trials, controlled before and after and controlled interrupted time series studies.

- Context: This was a broad review that was not restricted to any geographical location. However, this article includes only filtered studies conducted in LMICs as defined by the World Bank Gross National Income per capita, calculated using the World Bank Atlas method as of June 2019.

- Language: This review was restricted to articles published in the English language.

- Other considerations: We included only studies that had a sample size of at least 150 participants, a follow-up period of at least nine months and a participant attrition rate of less than $40 \%$ to minimize bias from included studies. We also excluded duplicate publications, systematic or narrative reviews, reviews, abstracts, letters to the editor, comments, case reports, conference presentations and study protocols. 


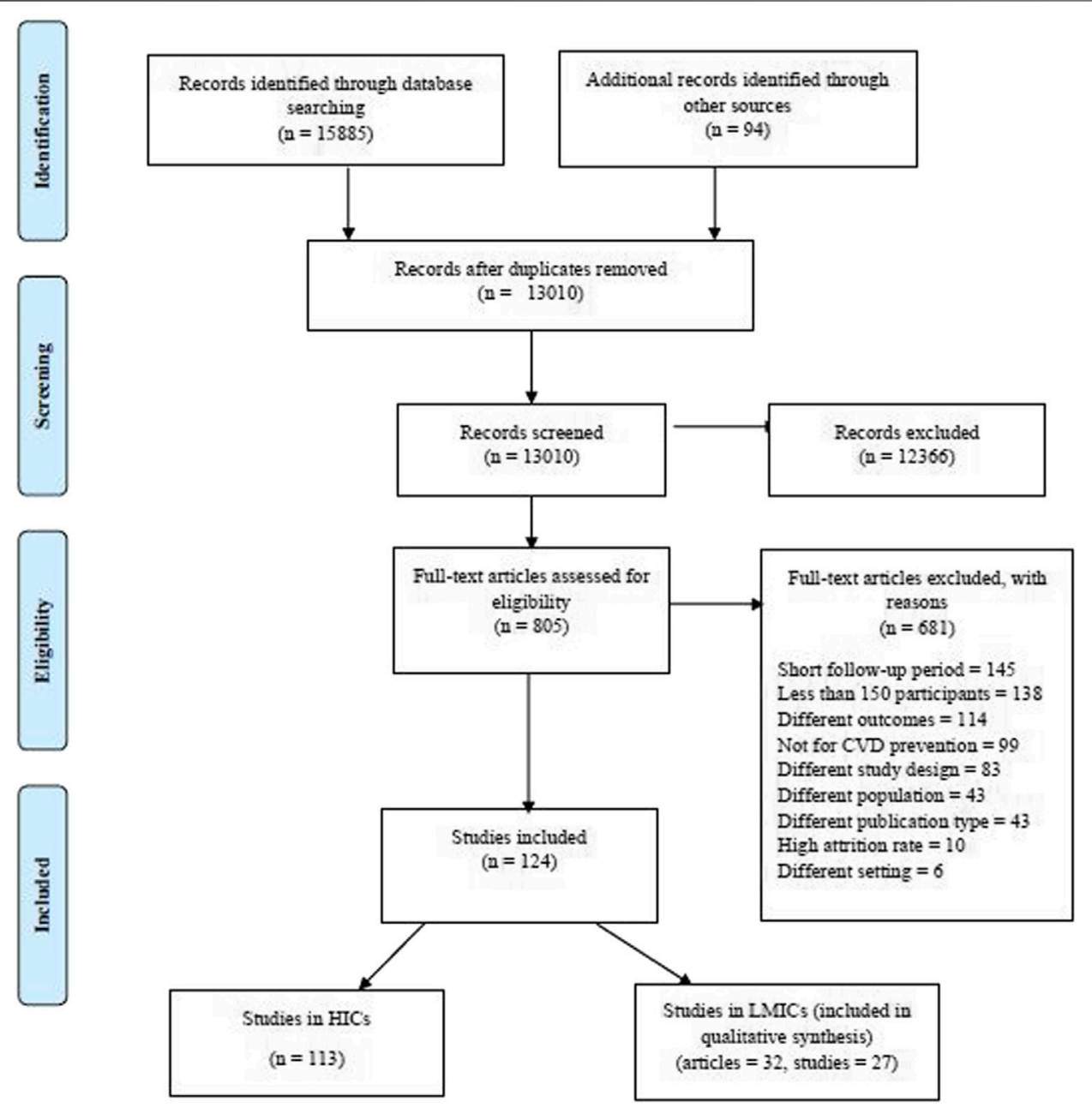

FIGURE 1 | PRISMA flow chart illustrating the article screening process, Community-based interventions for cardiovascular disease prevention in low- and middleincome countries: a systematic review, low- and middle-income countries, 2000-2019.

\section{Information Sources and Search Strategy} We searched MEDLINE, EMBASE, CINAHL, Cochrane register of controlled studies and PSYCINFO. Other sources of publications including thesis online, OpenGrey, ProQuest, Google Scholar and the World Health Organization (WHO) International Clinical Trials registry platform were searched. In addition, we searched reference lists of included studies and similar systematic reviews for potential eligible studies to include in this review. A comprehensive search strategy relating to the population, intervention, and outcomes was developed in MEDLINE (S2 Search strategy) and adjusted to suit other databases. Appropriate limits were applied and only studies conducted between January 2000 to June 2019 were retrieved. The search was however repeated before submission of the systematic review article to include any newer articles. Articles from all comprehensive searches of databases and gray literature and those obtained from reference lists of other articles were exported as EndNote files (including titles and abstracts) and then imported into EndNote as a single library. Duplicate articles from the searches were verified and removed. The remaining articles were imported into rayyan.QCRI.org [21], a web-based tool that facilitates screening and collaboration among researchers, for screening.

\section{Screening and Data Extraction}

Screening was conducted at two levels, title and abstract and full text independently in rayyan. QCRI by two reviewers ( $\mathrm{RN}$ and $\mathrm{HH}$ ) using defined criteria and in case of any disagreements, a third reviewer (HB or GM) made the final decision. We contacted authors by email in case of any key missing information in the articles. All reasons for exclusion of articles were noted and the review process was summarized within the PRISMA flow chart (Figure 1) highlighting the process of screening articles [22]. Data extraction was also independently conducted by two of the authors ( $\mathrm{RN}$ and $\mathrm{HH}$ ), thereby extracting all relevant information from included full text articles into a standardized Excel spreadsheet and later comparing and resolving any discrepancies. Data were extracted on study and participant characteristics, context, study design, methodology, intervention characteristics, comparator group(s) and outcome 
measures. For the outcomes, any effect estimates and observed changes in knowledge about CVDs, uptake of physical activity and diet, or reduction in smoking and alcohol use were recorded and data on secondary outcomes also extracted. Results of studies presented in multiple papers for the same population were only included in the review once.

\section{Risk of Bias Assessment}

The risk of bias of included studies was assessed using the revised Cochrane tool for Risk of Bias (RoB2) for randomized studies [23] and the Risk of Bias In Non-randomized Studies-of Interventions (ROBINS-I) for non-randomized studies [24]. The risk of bias assessment was conducted independently by two reviewers ( $\mathrm{RN}$ and $\mathrm{HH}$ ) who resolved any differences through consensus and if necessary, after consultation with a third reviewer (HB or GM).

\section{Strategy for Data Synthesis}

Data for this review were synthesized narratively while answering the aforementioned research questions. Findings have been descriptively presented and discussed while elaborating about the interventions and primary and secondary outcomes. Data have been presented in tabular form for comparison highlighting country, year of study, study objective, intervention, context, population and outcomes among others.

\section{RESULTS}

\section{Search Results}

Databases searched for this review yielded 15,885 articles. On top of that, 94 articles were identified through gray literature and snowball searching. At title and abstract screening, we retained 805 articles that underwent full text screening yielding 124 articles that met the inclusion criteria. Of the 124 articles, 32 were from LMICs representing 27 studies and were included in this review. A flow chart including details of the article screening process is shown in Figure 1.

\section{Characteristics of Included Studies Study Setting}

Of the 32 articles from which data were extracted, five were from the Isfahan Healthy Heart Program [25-29] and two from The Tehran Lipid and Glucose Study [30, 31] both conducted in Iran, which are reported using the most recent reference as [26, 30] respectively. Thus, this review includes a total of 27 studies as five articles were secondary references. Fourteen studies were from lower-middle [32-45] and eleven from upper-middle income countries [26, 30,46-54] or both [55] and only one was from a low-income country [56]. One study was conducted in Kenya in sub-Saharan Africa [32] and another in three countries, China, India and Mexico [55]. Of the remaining studies, seven were from India [33-37, 43, 55], five from China [46-50], and two from Iran [26, 30], Pakistan [38, 39] and Sri Lanka [42, 44]. Bangladesh [40], Grenada [54], Malaysia [51], Nepal [56], Thailand [53], Russia [52] and Vietnam [41] each had a single study.

\section{Study Designs and Context}

Nine studies were cluster randomized controlled trials [33, 34, 39, $40,42-45,56]$, eight were randomized controlled trials [35-37, $46,48,50,53,54]$ and ten were controlled before and after studies $[26,30,32,38,41,47,49,51,52,55]$. Studies were carried out in different contexts; eight in urban [30, 32, 36, 37, 39, 48, 50, 56], three in semi-urban $[42,44,51]$, eight in rural $[33,35,40,41,43$, $45,49,53]$ and two in both rural and urban areas [26, 38]. In six studies, the context was unclear [34, 46, 47, 52, 54, 55].

\section{Risk of Bias}

Among the included studies, the risk of bias categorization was: low (7), moderate (7), some concerns (9) and high/serious (4). The major sources of bias within included studies were: outcome measurement bias due to limited use of objective measures or validated tools, missing data bias due to omission of missing data or not applying appropriate analytical techniques, and the lack of control for some confounders for non randomized studies. Figures 2-4 show the risk of bias graphs and their summary drawn using the visualization tools by McGuinness and Higgins [57]. Overall, the randomized controlled and cluster randomized controlled trials had a lower risk of bias compared to the controlled before and after studies.

\section{Study Population}

Most studies targeted whole populations [26, 30, 32, 33, 38, $40-45,47,49,55,56]$ with health promotion and disease prevention activities while a few targeted risk groups including the elderly $[48,50]$, smokers $[35,52]$, individuals at high risk of diabetes [34, 36, 37, 51], those with hypertension [39, 46, 53] or more than one CVD risk factor [54]. All studies targeted both males and females except for four, three conducted in India, that targeted only males [35-37], and one in Sri Lanka that targeted mothers [54]. The age of participants in the studies was from 18 years and above and participant numbers ranged from 297 in Malaysia [51] to 12,514 in Iran [26].

\section{Variety of Community-Based Interventions}

The community-based interventions involved health education and awareness creation through mass media, mobile phones as well as information, communication and education materials $[26,30,32$, 33, 35-41, 45-47, 49, 50, 55]; trainings through workshops, lectures and small groups [26, 30, 38, 39, 48-51, 56]; lifestyle consultation and counseling either individually or in groups [33, 35-37, 39, 48, 51, 53, 56]; and community mobilization activities through meetings, peer support programmes and competitions [26, 30, $32,34,40,41,45,47,51,52,55]$. The other interventions were: environmental and structural changes in policies, infrastructure or institution of restrictions [26, 30, 47, 55]; and screening and treatment of risk factors [26, 32, 36, 41, 42, 48, 53, 56]. All studies used more than one strategy and most involved sharing information, health education, provision of community services and social mobilization. The least used strategies were changes in organisational culture and health policy and enforcement.

The intervention were delivered by healthcare workers $[26,30$, $32,36,39,41,47-50,52,53]$, community health workers, peers and volunteers [26, 30, 32-34, 38-40, 42, 53], local leaders and 

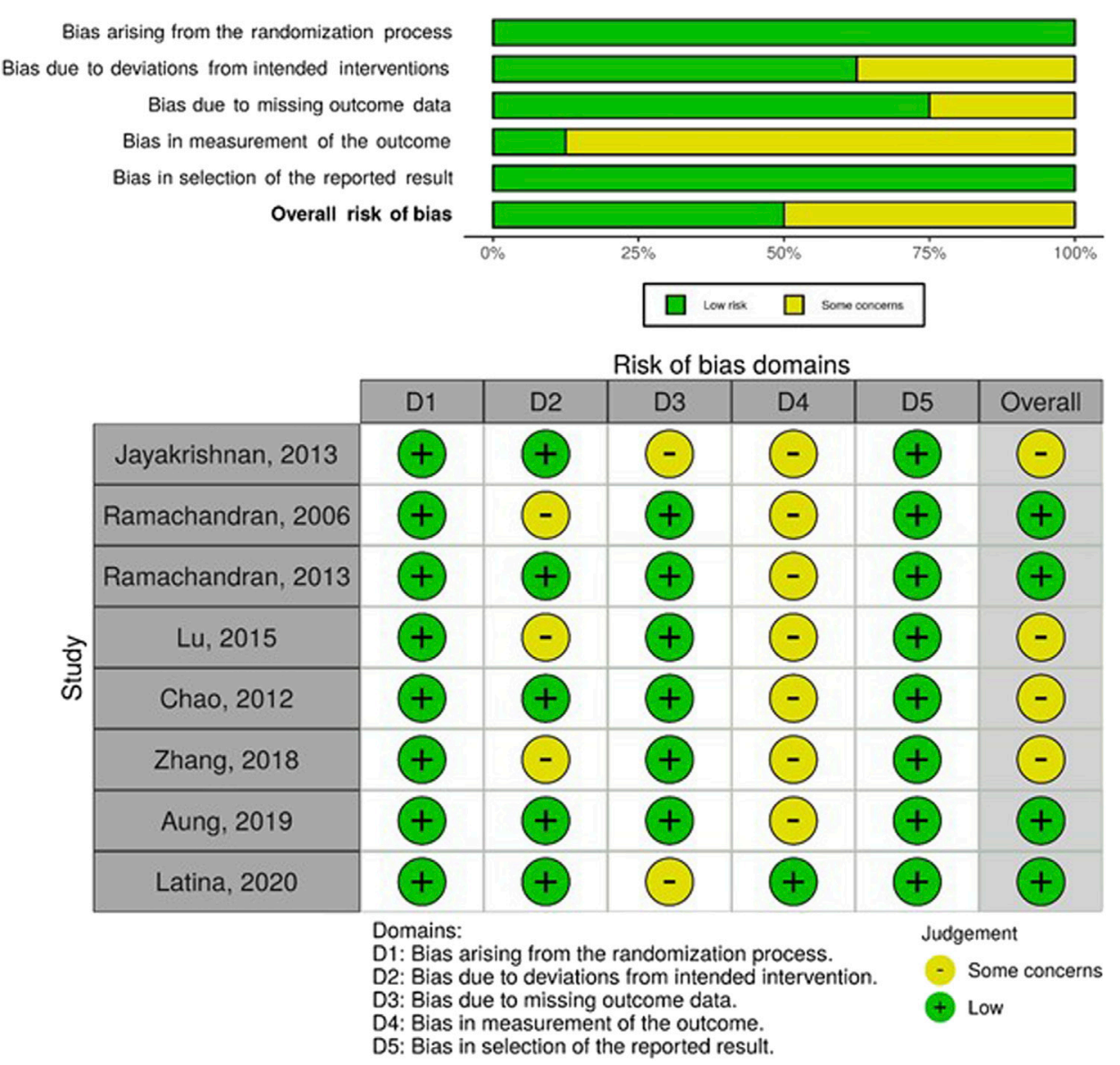

FIGURE 2 | Risk of bias graph (above) and summary (below); authors' judgements about each risk of bias item presented as percentages across all included randomized controlled trials, Community-based interventions for cardiovascular disease prevention in low- and middle-income countries: a systematic review, low- and middle-income countries, 2000-2019

resource persons [26, 30, 38, 47, 55], and researchers and experts [26, 33-35, 37, 46, 48, 49, 51]. The intervention settings were community $[26,30,32-42,45,49,51,52,55,56]$, community health care facilities [26, 30, 47, 50], schools [26, 30, 47, 55], workplaces [47, 55], neighbourhoods [47], and churches and mosques $[26,30]$. The interventions lasted between 6 months [35, 53] and 5 years [26] while the follow-up period ranged from 1 year $[33,35,42,51-53,56]$ to 5 years [26]. In five of the studies, the control group received a mild intervention [33-35, 37, 51] while for the rest, it was usual care or no intervention (Table 1).

\section{Effectiveness of Community-Based Interventions for CVD Prevention}

Table 2 summarizes the effect of the intervention on the behavioral and metabolic outcomes.

\section{Primary Outcomes}

\section{Knowledge on CVDs and Risk Factors}

Six studies half of which had moderate/some concerns RoB [ 40 , $46,47]$ and the rest had a high/serious $\operatorname{RoB}[38,45,49]$ examined changes in CVD knowledge following implementation of community-based interventions. In five of the studies, knowledge significantly improved in the intervention groups related to dietary and lifestyle factors [38, 47, 49], hypertension [38, 46, 49] and diabetes [40] compared to the control groups. In another study in China, although tobacco related knowledge increased, diet and physical activity knowledge decreased in the intervention compared to the control group [47]. In a study with a high RoB carried out in India, there was no statistically significant effect of the intervention on knowledge about the six lifestyle factors affecting CVD risk [45]. Interventions that were effective in enhancing CVD knowledge majorly involved training, community mobilization, health education and consultation delivered through campaigns, group meetings, workshops, use of mobile technologies and of health workers, community health workers or peers.

\section{Physical Activity}

A total of 23 studies recorded changes in physical activity across study populations. Among these, 16 studies compared improvements in physical activity between the intervention 


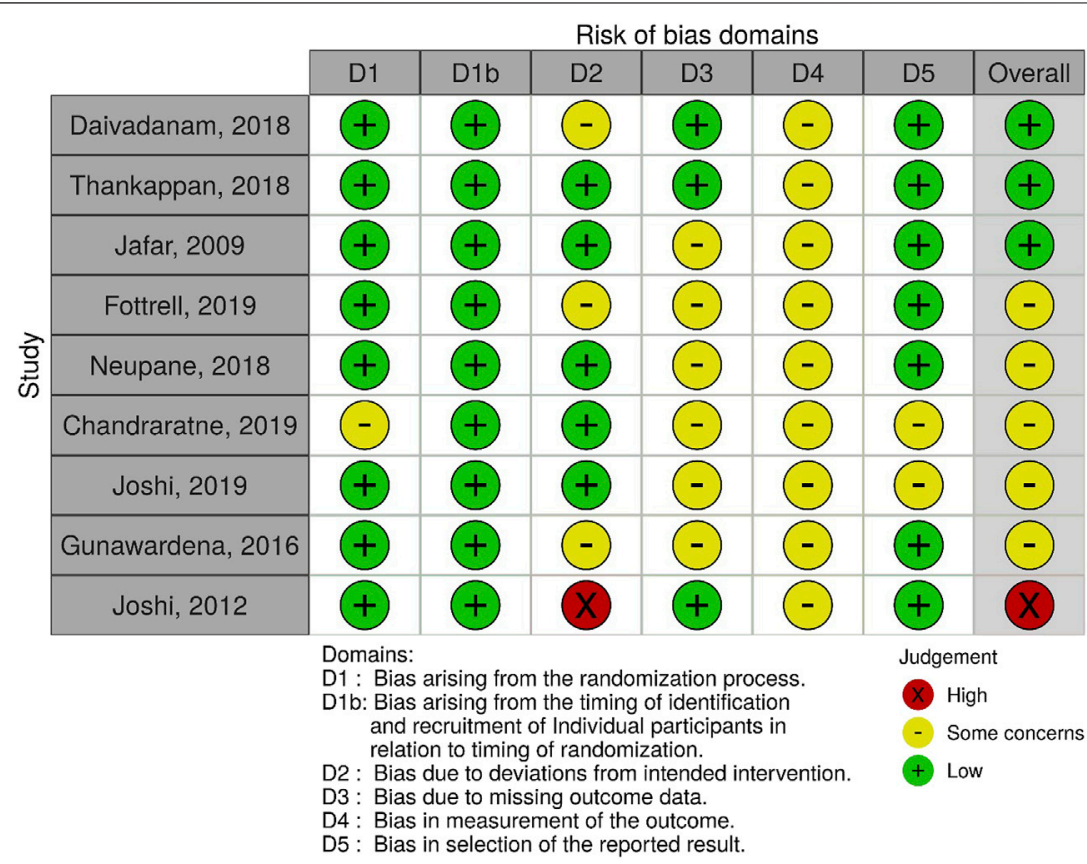

FIGURE 3 | Risk of bias summary; authors' judgements about each risk of bias item for each included cluster randomized controlled trial, Community-based interventions for cardiovascular disease prevention in low- and middle-income countries: a systematic review, low- and middle-income countries, $2000-2019$.

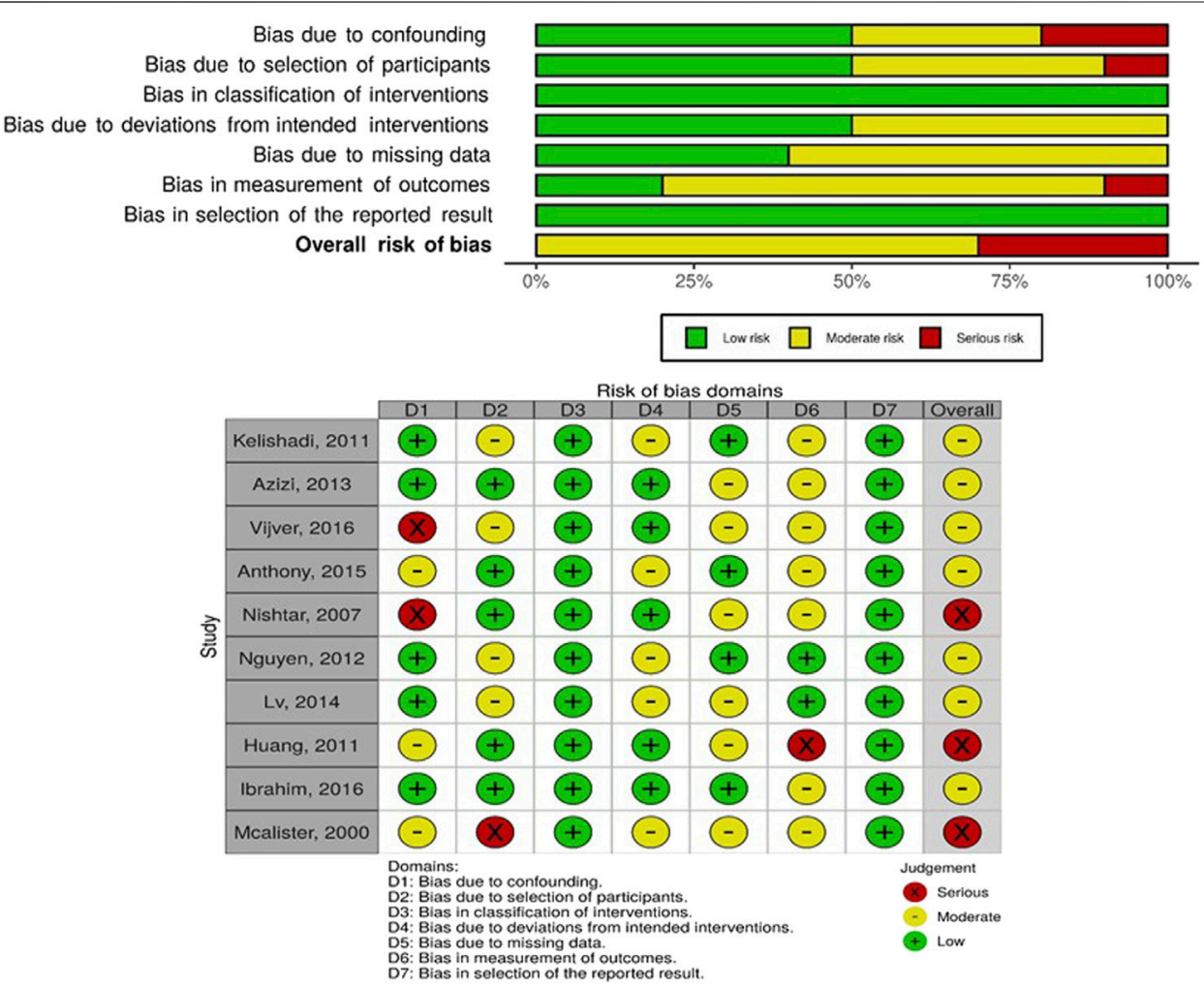

FIGURE 4 | Risk of bias graph (above) and summary (below); authors' judgements about each risk of bias item presented as percentages across all included controlled before and after studies, Community-based interventions for cardiovascular disease prevention in low- and middle-income countries: a systematic review, low- and middle-income countries, 2000-2019. 
TABLE 1 | Characteristics of included studies and community-based interventions, Community-based interventions for cardiovascular disease prevention in low- and middle-income countries: a systematic review, 2000-2019.

\begin{tabular}{|c|c|c|c|c|c|c|c|c|c|c|c|c|c|}
\hline Author & $\begin{array}{l}\text { Country } \\
\text { (income } \\
\text { category) }\end{array}$ & $\begin{array}{l}\text { Study } \\
\text { design }\end{array}$ & $\begin{array}{c}\text { Study } \\
\text { aim }\end{array}$ & Context & $\begin{array}{c}\text { Target } \\
\text { population }\end{array}$ & Age & Participants & Intervention & Length & Provider & Setting & $\begin{array}{c}\text { Follow-up } \\
\text { time }\end{array}$ & Control \\
\hline $\begin{array}{l}\text { Baghaei, } \\
2010 \text { [25] }\end{array}$ & $\begin{array}{l}\text { Iran } \\
\text { (upperMIC) }\end{array}$ & $\begin{array}{l}\text { Controlled } \\
\text { before and } \\
\text { after }\end{array}$ & $\begin{array}{l}\text { To demonstrate } \\
\text { the efficacy of the } \\
\text { isfahan healthy } \\
\text { heart program } \\
\text { interventional } \\
\text { strategies to } \\
\text { improve lifestyle } \\
\text { behaviors in a } \\
\text { population at risk } \\
\text { for developing } \\
\text { cardiovascular } \\
\text { diseases }\end{array}$ & $\begin{array}{l}\text { Urban and rural } \\
\text { isafayan (intervention) } \\
\text { and najafabad } \\
\text { (reference) }\end{array}$ & $\begin{array}{l}\text { Adults with } \\
\text { atleast one CVD } \\
\text { risk factors } \\
\text { including high } \\
\text { blood pressure, } \\
\text { diabetes } \\
\text { mellitus, } \\
\text { dyslipidaemia, } \\
\text { metabollic } \\
\text { syndrome, } \\
\text { obesity and } \\
\text { smoking }\end{array}$ & $\begin{array}{l}19 \text { years } \\
\text { and above }\end{array}$ & 9,411 & $\begin{array}{l}\text { 1) policy/Environmental } \\
\text { strategies. 2) community } \\
\text { outreach/Program services } \\
\text { and. 3) survillance }\end{array}$ & 5 years & $\begin{array}{l}\text { Mass media, } \\
\text { campaigns }\end{array}$ & Community & 5 years & No intervention \\
\hline $\begin{array}{l}\text { Kelishadi, } \\
2011 \text { [26] }\end{array}$ & $\begin{array}{l}\text { Iran } \\
\text { (upperMIC) }\end{array}$ & $\begin{array}{l}\text { Controlled } \\
\text { before and } \\
\text { after }\end{array}$ & $\begin{array}{l}\text { To investigate the } \\
\text { effect of a } \\
\text { comprehensive } \\
\text { community trial on } \\
\text { behavioral } \\
\text { modification after } \\
2 \text { years of } \\
\text { intervention }\end{array}$ & $\begin{array}{l}\text { Urban and rural areas } \\
\text { (two cities for } \\
\text { intervention and one for } \\
\text { control) }\end{array}$ & $\begin{array}{l}\text { Adults in } \\
\text { selected cities }\end{array}$ & $\begin{array}{l}19 \text { years } \\
\text { and above }\end{array}$ & 12,514 & $\begin{array}{l}\text { Community mobilization } \\
\text { through training the trainers, } \\
\text { activities to improve } \\
\text { knowledge, attitude and } \\
\text { practice, sport and physical } \\
\text { activity, education through } \\
\text { social gatherings, and } \\
\text { involvement of community } \\
\text { leaders }\end{array}$ & 2 years & $\begin{array}{l}\text { Mass media, health } \\
\text { professionals and } \\
\text { market leaders, key } \\
\text { nongovernmental } \\
\text { organization staff, } \\
\text { and local political } \\
\text { decision makers } \\
\text { (county, municipal, } \\
\text { and provincial } \\
\text { leaders) }\end{array}$ & Community & 2 years & No intervention \\
\hline Rabiei, 2010 [27] & $\begin{array}{l}\text { Iran } \\
\text { (upperMIC) }\end{array}$ & $\begin{array}{l}\text { Controlled } \\
\text { before and } \\
\text { after }\end{array}$ & $\begin{array}{l}\text { To present the } \\
\text { changes in PA } \\
\text { habits after } \\
2 \text { years of } \\
\text { intervention for } \\
\text { increasing PA. }\end{array}$ & $\begin{array}{l}\text { Urban and rural areas } \\
\text { of isfahan and } \\
\text { najafababad }\end{array}$ & $\begin{array}{l}\text { Adults in } \\
\text { selected cities }\end{array}$ & $\begin{array}{l}19 \text { years } \\
\text { and above }\end{array}$ & 12,514 & $\begin{array}{l}\text { Educational interventions-face } \\
\text { to face or through class or } \\
\text { education materials and } \\
\text { camapigns. Environmental } \\
\text { interventions-infrastructures } \\
\text { for cycling, walking and use of } \\
\text { public transportation. } \\
\text { Legislative interventions-rules } \\
\text { around physical activity in } \\
\text { schools and directives on } \\
\text { exercise time }\end{array}$ & 2 years & $\begin{array}{l}\text { Mass media, health } \\
\text { professionals and } \\
\text { market leaders, key } \\
\text { nongovernmental } \\
\text { organization staff, } \\
\text { and local political } \\
\text { decision makers } \\
\text { (county, municipal, } \\
\text { and provincial } \\
\text { leaders) }\end{array}$ & Community & 2 years & No intervention \\
\hline $\begin{array}{l}\text { Sarrafzadegan, } \\
2009 \text { [28] }\end{array}$ & $\begin{array}{l}\text { Iran } \\
\text { (upperMIC) }\end{array}$ & $\begin{array}{l}\text { Controlled } \\
\text { before and } \\
\text { after }\end{array}$ & $\begin{array}{l}\text { To study the } \\
\text { feasibility and } \\
\text { impact of a } \\
\text { comprehensive, } \\
\text { integrated, } \\
\text { community-based } \\
\text { program directed } \\
\text { toward reducing } \\
\text { modififable risk } \\
\text { factors for } \\
\text { cardiovascular } \\
\text { disease }\end{array}$ & Urban and rural areas & $\begin{array}{l}\text { General } \\
\text { population, the } \\
\text { high-risk groups } \\
\text { as well as } \\
\text { specific target } \\
\text { groups in urban } \\
\text { and rural areas } \\
\text { of the } \\
\text { intervention. } \\
\text { communities } \\
\text { (isfahan and } \\
\text { najaf-abad) }\end{array}$ & $\begin{array}{l}19 \text { years } \\
\text { and above }\end{array}$ & 12,514 & $\begin{array}{l}\text { Radio and television series for } \\
\text { a healthy diet, exercise, } \\
\text { educational cartoons and films } \\
\text { for children, etc.,. different } \\
\text { smoking cessation campaigns } \\
\text { like the international smoking } \\
\text { cessation program "quit } \\
\text { andWWin" or big campaigns } \\
\text { about passive smoking, } \\
\text { healthy lifestyle }\end{array}$ & 5 years & $\begin{array}{l}\text { Multiple including } \\
\text { mass media, } \\
\text { community health } \\
\text { profossionals, role } \\
\text { models and opinion } \\
\text { leaders, religious } \\
\text { leaders etc. }\end{array}$ & Community & 5 years & No intervention \\
\hline $\begin{array}{l}\text { Sarrafzadegan, } \\
2009[29]\end{array}$ & $\begin{array}{l}\text { Iran } \\
\text { (upperMIC) }\end{array}$ & $\begin{array}{l}\text { Controlled } \\
\text { before and } \\
\text { after }\end{array}$ & $\begin{array}{l}\text { To assess the } \\
\text { effects of a } \\
\text { comprehensive, } \\
\text { integrated } \\
\text { community-based } \\
\text { lifestyle } \\
\text { intervention on } \\
\text { diet, physical } \\
\text { activity and } \\
\text { smoking in two } \\
\text { iranian } \\
\text { communities }\end{array}$ & Urban and rural areas & $\begin{array}{l}\text { General } \\
\text { population as } \\
\text { well as specific } \\
\text { groups within } \\
\text { the intervention } \\
\text { communities }\end{array}$ & $\begin{array}{l}19 \text { years } \\
\text { and above }\end{array}$ & 12,514 & $\begin{array}{l}\text { Public education through the } \\
\text { mass media, inter-sectoral } \\
\text { cooperation and collaboration, } \\
\text { community participation, } \\
\text { education and involvement of } \\
\text { health professionals, } \\
\text { marketing and organizational } \\
\text { development, legislation and } \\
\text { policy development or } \\
\text { enforcement, and research } \\
\text { and evaluation }\end{array}$ & 4 years & Multiple & $\begin{array}{l}\text { Community and } \\
\text { health facility }\end{array}$ & 4 years & No intervention \\
\hline
\end{tabular}


TABLE 1 | (Continued) Characteristics of included studies and community-based interventions, Community-based interventions for cardiovascular disease prevention in low- and middle-income countries: a systematic review, 2000-2019.

\begin{tabular}{|c|c|c|c|c|c|c|c|c|c|c|c|c|c|}
\hline Author & $\begin{array}{l}\text { Country } \\
\text { (income } \\
\text { category) }\end{array}$ & $\begin{array}{l}\text { Study } \\
\text { design }\end{array}$ & $\begin{array}{c}\text { Study } \\
\text { aim }\end{array}$ & Context & $\begin{array}{c}\text { Target } \\
\text { population }\end{array}$ & Age & Participants & Intervention & Length & Provider & Setting & $\begin{array}{l}\text { Follow-up } \\
\text { time }\end{array}$ & Control \\
\hline Azizi, 2013 [30] & $\begin{array}{l}\text { Iran } \\
\text { (upperMIC) }\end{array}$ & $\begin{array}{l}\text { Controlled } \\
\text { before and } \\
\text { after }\end{array}$ & $\begin{array}{l}\text { To assess the } \\
\text { effects of lifestyle } \\
\text { modifications on } \\
\text { metabolic } \\
\text { syndrome and } \\
\text { some of its } \\
\text { components in } \\
\text { urban population } \\
\text { of the lipids and } \\
\text { glucose study }\end{array}$ & $\begin{array}{l}\text { Urban residents of } \\
\text { district } 13 \text { within } \\
\text { tehran, the capital of } \\
\text { Iran }\end{array}$ & $\begin{array}{l}\text { Individuals who } \\
\text { had returned for } \\
\text { follow up after } \\
3.6 \text { years }\end{array}$ & $20-74$ years & 6,870 & $\begin{array}{l}\text { Nutrition education classes; } \\
\text { healthy nutrition messages } \\
\text { written in health newsletter. } \\
\text { Distribution of pamphlets, } \\
\text { brochures and booklets } \\
\text { written on smoking, nutrtition, } \\
\text { physical activity and coping } \\
\text { with stress. Nutrtition } \\
\text { intervention in nutrtition clinics } \\
\text { for subjects with diseases such } \\
\text { as diabetes, voreaguight and } \\
\text { obesity, dyslipidemia and } \\
\text { hypertension }\end{array}$ & 3.6 years & $\begin{array}{l}\text { Health volunteers } \\
\text { and clinic staff }\end{array}$ & $\begin{array}{l}\text { Community, } \\
\text { school and facility } \\
\text { based }\end{array}$ & 3.6 years & Not clear \\
\hline $\begin{array}{l}\text { Mirmiran, } \\
2008[31]\end{array}$ & $\begin{array}{l}\text { Iran } \\
\text { (upperMIC) }\end{array}$ & $\begin{array}{l}\text { Controlled } \\
\text { before and } \\
\text { after }\end{array}$ & $\begin{array}{l}\text { To determine the } \\
\text { effectiveness of } \\
\text { nutrition } \\
\text { intervention on } \\
\text { non- } \\
\text { communicable } \\
\text { disease risk } \\
\text { factors among } \\
\text { tehranian urban } \\
\text { adults }\end{array}$ & $\begin{array}{l}\text { Residents in an urban } \\
\text { distrct of Iran }\end{array}$ & $\begin{array}{l}\text { Subjects aged } \\
3 \text { years and } \\
\text { above }\end{array}$ & $18-74$ years & 578 & $\begin{array}{l}\text { Nutrition intervention } \\
\text { introduced for all individuals } \\
\text { aged } 3 \text { years and over in } \\
\text { health care centers, schools } \\
\text { and public places. At health } \\
\text { facilities, family members } \\
\text { invited and trained with face to } \\
\text { face contact between } \\
\text { educators and participants. In } \\
\text { schools education by } \\
\text { teachers, cooperation } \\
\text { societies and group based } \\
\text { activities including fairs and } \\
\text { competitions. Foods at school } \\
\text { canteen changed according to } \\
\text { guidleines }\end{array}$ & 3.8 years & $\begin{array}{l}\text { Educators at health } \\
\text { facilities and trained } \\
\text { teachers, parent- } \\
\text { teacher cooperation } \\
\text { societies, and } \\
\text { group-based } \\
\text { activities such as } \\
\text { fairs and } \\
\text { competitions in } \\
\text { schools }\end{array}$ & $\begin{array}{l}\text { Community, health } \\
\text { care centers, } \\
\text { schools, and } \\
\text { public places }\end{array}$ & 3.8 years & $\begin{array}{l}\text { No nutrition } \\
\text { interventions }\end{array}$ \\
\hline Vijver, 2016 [32] & $\begin{array}{l}\text { Kenya } \\
\text { (lower MIC) }\end{array}$ & $\begin{array}{l}\text { Controlled } \\
\text { before and } \\
\text { after }\end{array}$ & $\begin{array}{l}\text { To evaluate the } \\
\text { impact of a } \\
\text { community-based } \\
\text { CVD prevention } \\
\text { intervention on } \\
\text { blood pressure } \\
\text { (BP) and other } \\
\text { CVD risk factors in } \\
\text { a slum setting in } \\
\text { nairobi, Kenya }\end{array}$ & $\begin{array}{l}\text { Urban poor living in two } \\
\text { slums, korogocho and } \\
\text { viwandani }\end{array}$ & $\begin{array}{l}\text { Adults living in } \\
\text { the slums }\end{array}$ & $\begin{array}{l}35 \text { years } \\
\text { and above }\end{array}$ & 2,764 & $\begin{array}{l}\text { Raising awareness through } \\
\text { mass media and public places, } \\
\text { improving access to screening } \\
\text { through BP measurement by } \\
\text { community health workers, } \\
\text { facilitating access to treatment } \\
\text { through providing vouchers, } \\
\text { and promoting long-term } \\
\text { retention in care through } \\
\text { encouraging visits, patient } \\
\text { support groups and providing } \\
\text { subsidies }\end{array}$ & 18 months & $\begin{array}{l}\text { Radio, CHWs, } \\
\text { health workers }\end{array}$ & Community & 18 months & $\begin{array}{l}\text { Access to CVD } \\
\text { standard of care } \\
\text { (access outside } \\
\text { the slum) }\end{array}$ \\
\hline $\begin{array}{l}\text { Daivadanam, } \\
2018 \text { [33] }\end{array}$ & $\begin{array}{l}\text { India } \\
\text { (lower MIC) }\end{array}$ & $\begin{array}{l}\text { Cluster } \\
\text { randomized } \\
\text { controlled trial }\end{array}$ & $\begin{array}{l}\text { To test the } \\
\text { effectiveness of a } \\
\text { sequential stage- } \\
\text { matched } \\
\text { interention } \\
\text { strategy to } \\
\text { increase the daily } \\
\text { intake of fruits and } \\
\text { vegetables by an } \\
\text { absolute } 20 \% \\
\text { from baseline in } \\
\text { the intervention } \\
\text { arm over a one- } \\
\text { year intervention } \\
\text { period }\end{array}$ & Rural India & $\begin{array}{l}\text { Adult males or } \\
\text { females in the } \\
\text { geographical } \\
\text { area }\end{array}$ & $25-45$ years & 479 & $\begin{array}{l}\text { Initial face-to-face counseling, } \\
\text { home-visits, general } \\
\text { awareness sessions for } \\
\text { community members in } \\
\text { groups. Sequential stage- } \\
\text { matching to differentiate } \\
\text { households based on } \\
\text { readiness to change dietary } \\
\text { behavior in order to determine } \\
\text { the strategies to be delivered to } \\
\text { each household }\end{array}$ & 9 months & $\begin{array}{l}\text { Counselors and } \\
\text { psychology } \\
\text { students trained in } \\
\text { brief intervention. } \\
\text { Community } \\
\text { volunteers trained to } \\
\text { deliver specific } \\
\text { strategies and study } \\
\text { coordinator }\end{array}$ & Community & 12 months & $\begin{array}{l}\text { Information on } \\
\text { recommended } \\
\text { levels of intake of } \\
\text { the five dietary } \\
\text { components, } \\
\text { and general } \\
\text { dietary } \\
\text { information } \\
\text { leaflets }\end{array}$ \\
\hline
\end{tabular}


TABLE 1 ( (Continued) Characteristics of included studies and community-based interventions, Community-based interventions for cardiovascular disease prevention in low- and middle-income countries: a systematic review, 2000-2019.

\begin{tabular}{|c|c|c|c|c|c|c|c|c|c|c|c|c|c|}
\hline Author & $\begin{array}{l}\text { Country } \\
\text { (income } \\
\text { category) }\end{array}$ & $\begin{array}{l}\text { Study } \\
\text { design }\end{array}$ & $\begin{array}{c}\text { Study } \\
\text { aim }\end{array}$ & Context & $\begin{array}{c}\text { Target } \\
\text { population }\end{array}$ & Age & Participants & Intervention & Length & Provider & Setting & $\begin{array}{l}\text { Follow-up } \\
\text { time }\end{array}$ & Control \\
\hline $\begin{array}{l}\text { Thankappan, } \\
2018 \text { [34] }\end{array}$ & $\begin{array}{l}\text { India } \\
\text { (lower MIC) }\end{array}$ & $\begin{array}{l}\text { Cluster } \\
\text { randomized } \\
\text { trial }\end{array}$ & $\begin{array}{l}\text { To evaluate the } \\
\text { effectiveness of a } \\
\text { peer-support } \\
\text { lifestyle } \\
\text { intervention in } \\
\text { preventing type } 2 \\
\text { diabetes among } \\
\text { high-risk } \\
\text { individuals } \\
\text { identified on the } \\
\text { basis of a simple } \\
\text { diabetes risk score }\end{array}$ & $\begin{array}{l}\text { Neighboorhoods in } \\
\text { close proximity }\end{array}$ & $\begin{array}{l}\text { Individuals with } \\
\text { an indian } \\
\text { diabetes risk } \\
\text { score } \geq 60 \text { and } \\
\text { free from } \\
\text { diabetes on an } \\
\text { oral glucose } \\
\text { tolerence test }\end{array}$ & $30-60$ years & 1,007 & $\begin{array}{l}\text { Community-based peer- } \\
\text { support program, a range of } \\
\text { group session and community } \\
\text { activities to support lifestyle } \\
\text { change on diabetes and risk } \\
\text { factors, nutiriton and physical } \\
\text { activity held in community } \\
\text { facilities such as schools and } \\
\text { community halls and peer } \\
\text { group sessions were held } \\
\text { monthly except for the first two } \\
\text { done fortnightly }\end{array}$ & 12 months & $\begin{array}{l}\text { Diabetes and } \\
\text { lifestyle experts and } \\
\text { trained peer leaders }\end{array}$ & Community & 24 months & $\begin{array}{l}\text { An education } \\
\text { booklet with } \\
\text { lifestyle change } \\
\text { advice }\end{array}$ \\
\hline $\begin{array}{l}\text { Jayakrishnan, } \\
2013[35]\end{array}$ & $\begin{array}{l}\text { India } \\
\text { (lower MIC) }\end{array}$ & $\begin{array}{l}\text { Randomized } \\
\text { controlled trial }\end{array}$ & $\begin{array}{l}\text { To assess the } \\
\text { effectiveness of a } \\
\text { cessation } \\
\text { intervention in rural } \\
\text { Kerala state, India }\end{array}$ & $\begin{array}{l}4 \text { randomly allocated. } \\
\text { Community } \\
\text { development blocks in } \\
\text { rural. } \\
\text { Thiruvananthapuram } \\
\text { district of Kerala state in } \\
\text { south India }\end{array}$ & $\begin{array}{l}\text { Current daily } \\
\text { smoking } \\
\text { resident males }\end{array}$ & $18-60$ years & 928 & $\begin{array}{l}\text { Awareness on tobacco } \\
\text { hazards, anti-tobacco leaflets, } \\
\text { a "how to quit tobacco" guide } \\
\text { and a quick reference guide } \\
\text { titled "how to quit tobacco". } \\
\text { Group counseling and face-to- } \\
\text { face individual counseling for } \\
\text { participants through house } \\
\text { visits or mobile phones }\end{array}$ & 6 months & $\begin{array}{l}\text { Medical social } \\
\text { workers }\end{array}$ & Community & 12 months & $\begin{array}{l}\text { General } \\
\text { awareness } \\
\text { training on } \\
\text { tobacco hazards } \\
\text { along with an } \\
\text { anti-tobacco } \\
\text { leaflet. }\end{array}$ \\
\hline $\begin{array}{l}\text { Ramachandran, } \\
2006 \text { [36] }\end{array}$ & $\begin{array}{l}\text { India } \\
\text { (lower MIC) }\end{array}$ & $\begin{array}{l}\text { Randomized, } \\
\text { controlled trial }\end{array}$ & $\begin{array}{l}\text { To determine } \\
\text { whether the } \\
\text { incidence of type } 2 \\
\text { diabetes could be } \\
\text { modified by } \\
\text { interventions }\end{array}$ & $\begin{array}{l}\text { An urban population in } \\
\text { India }\end{array}$ & $\begin{array}{l}\text { Middle-class } \\
\text { working indian } \\
\text { men with } \\
\text { impared } \\
\text { glucose } \\
\text { tolerance }\end{array}$ & $35-55$ years & 502 & $\begin{array}{l}\text { Lifestyle modification group } \\
\text { including advice on healthy diet } \\
\text { and regular physical activity. } \\
\text { Metformin (MET) group with } \\
\text { diaries to record daily } \\
\text { consumption of tablets. LSM + } \\
\text { met group-subjects were } \\
\text { given LSM plus MET. All } \\
\text { intervention groups received } \\
\text { monthly phone calls for } \\
\text { continued motivation }\end{array}$ & 30 and 36 months & Health workers & Community & $\begin{array}{l}30 \text { and } \\
36 \text { months }\end{array}$ & $\begin{array}{l}\text { Standard health } \\
\text { care advice }\end{array}$ \\
\hline $\begin{array}{l}\text { Ramachandran, } \\
2013[37]\end{array}$ & $\begin{array}{l}\text { India } \\
\text { (lower MIC) }\end{array}$ & $\begin{array}{l}\text { Randomized } \\
\text { controlled trial }\end{array}$ & $\begin{array}{l}\text { To assess whether } \\
\text { mobile phone } \\
\text { messaging that } \\
\text { encouraged } \\
\text { lifestyle change } \\
\text { could reduce } \\
\text { incident type 2 } \\
\text { diabetes in indian } \\
\text { asian men with } \\
\text { impaired glucose } \\
\text { tolerance }\end{array}$ & $\begin{array}{l}\text { An urban population in } \\
\text { India }\end{array}$ & $\begin{array}{l}\text { Working indian } \\
\text { men with } \\
\text { impaired } \\
\text { glucose } \\
\text { tolerance }\end{array}$ & $35-55$ years & 537 & $\begin{array}{l}\text { Personalized education and } \\
\text { motivation about healthy } \\
\text { lifestyle principles and trequent } \\
\text { mobile phone messages-with } \\
\text { information about healthy } \\
\text { lifestyle, the benefits of physical } \\
\text { activity and diet, cues to start, } \\
\text { and strategies to avoid relapse }\end{array}$ & 2 years & $\begin{array}{l}\text { Study staff, mobile } \\
\text { phone delivery } \\
\text { website }\end{array}$ & Community & 3 years & $\begin{array}{l}\text { Standard lifestyle } \\
\text { modification } \\
\text { advice at } \\
\text { baseline only }\end{array}$ \\
\hline $\begin{array}{l}\text { Nishtar, } \\
2007[38]\end{array}$ & $\begin{array}{l}\text { Pakistan } \\
\text { (lower MIC) }\end{array}$ & $\begin{array}{l}\text { Controlled } \\
\text { before and } \\
\text { after }\end{array}$ & $\begin{array}{l}\text { To examine } \\
\text { changes in } \\
\text { knowledge and } \\
\text { CVD risk factors } \\
\text { among the } \\
\text { community } \\
\text { following an } \\
\text { intervention }\end{array}$ & $\begin{array}{l}85.5 \% \text { of district is rural } \\
\text { whereas } 14.5 \% \text { is } \\
\text { urban with high rates of } \\
\text { poverty, illteracy, low } \\
\text { education levels and } \\
\text { high unemployment }\end{array}$ & $\begin{array}{l}\text { Males and } \\
\text { females resident } \\
\text { in the district }\end{array}$ & $18-65$ years & 604 & $\begin{array}{l}\text { Community health education } \\
\text { during meetings through } \\
\text { organized groups, mass media } \\
\text { interventions, training of health } \\
\text { professionals through one-day } \\
\text { workshops and lady health } \\
\text { workers, and health education } \\
\text { through lady health workers } \\
\text { who undergo } 3 \text { days monthly } \\
\text { training }\end{array}$ & 2 years & $\begin{array}{l}\text { Trained officer for } \\
\text { health education, } \\
\text { radio, lady heath } \\
\text { workers }\end{array}$ & Community & 2 years & No intervention \\
\hline
\end{tabular}


TABLE 1 | (Continued) Characteristics of included studies and community-based interventions, Community-based interventions for cardiovascular disease prevention in low- and middle-income countries: a systematic review, 2000-2019.

\begin{tabular}{|c|c|c|c|c|c|c|c|c|c|c|c|c|c|}
\hline Author & $\begin{array}{l}\text { Country } \\
\text { (income } \\
\text { category) }\end{array}$ & $\begin{array}{l}\text { Study } \\
\text { design }\end{array}$ & $\begin{array}{l}\text { Study } \\
\text { aim }\end{array}$ & Context & $\begin{array}{c}\text { Target } \\
\text { population }\end{array}$ & Age & Participants & Intervention & Length & Provider & Setting & $\begin{array}{l}\text { Follow-up } \\
\text { time }\end{array}$ & Control \\
\hline Jafar, 2009 [39] & $\begin{array}{l}\text { Pakistan } \\
\text { (lower MIC) }\end{array}$ & $\begin{array}{l}\text { Cluster } \\
\text { randomized } \\
\text { controlled trial }\end{array}$ & $\begin{array}{l}\text { To assess the } \\
\text { effectiveness of } \\
\text { two community- } \\
\text { based } \\
\text { interventions on } \\
\text { blood pressure in } \\
\text { hypertensive } \\
\text { adults }\end{array}$ & $\begin{array}{l}\text { Households in karachi, } \\
\text { the most populous city } \\
\text { in Pakistan (mean } \\
\text { household monthly } \\
\text { income, \$70) }\end{array}$ & $\begin{array}{l}\text { Residents in the } \\
\text { area and had } \\
\text { known } \\
\text { hypertension or } \\
\text { consistently } \\
\text { elevated blood } \\
\text { pressure on } 2 \\
\text { separate visits }\end{array}$ & $\begin{array}{l}40 \text { years } \\
\text { and above }\end{array}$ & 1,341 & $\begin{array}{l}\text { Family based home heatth } \\
\text { education from lay health } \\
\text { workers and annual training of } \\
\text { general practitioners in } \\
\text { hypertension management. } \\
\text { HHE alone: Trained CHWs } \\
\text { deliver behavior changing } \\
\text { communication strategies to } \\
\text { convey standardized heaath } \\
\text { educatiton messages to } \\
\text { households. GP alone: } \\
\text { of at at leasining } \\
\text { in area. HHE and thirds of the GPs } \\
\text { aP combined }\end{array}$ & 2 years & $\begin{array}{l}\text { Lay health workers } \\
\text { and GPs }\end{array}$ & Community & 2 years & No intervention \\
\hline $\begin{array}{l}\text { Fottrell, } \\
2019[40]\end{array}$ & $\begin{array}{l}\text { Bangladesh } \\
\text { (lower MIC) }\end{array}$ & $\begin{array}{l}\text { Cluster } \\
\text { randomized } \\
\text { trial }\end{array}$ & $\begin{array}{l}\text { To assess the } \\
\text { effects of mHealth } \\
\text { and community } \\
\text { mobilization on the } \\
\text { prevalence of } \\
\text { intermediate } \\
\text { hyperglycaemia } \\
\text { and diabetes } \\
\text { among the general } \\
\text { adult population }\end{array}$ & Rural Bangladesh & $\begin{array}{l}\text { Men and non- } \\
\text { pregnant } \\
\text { women }\end{array}$ & $\begin{array}{l}30 \text { years } \\
\text { and above }\end{array}$ & $\begin{array}{l}12,280 \text { for } \\
\text { cross } \\
\text { sectional and } \\
2,470 \text { for } \\
\text { cohort }\end{array}$ & $\begin{array}{l}\text { Participatory community } \\
\text { mobilization-involved } \\
18 \text { monthly group meetings, } \\
\text { led by lay facilitators, applying a } \\
\text { participatory learning and } \\
\text { action (PLA) cycle focused on } \\
\text { diabetes prevention and } \\
\text { control. mHHeath mobile phone } \\
\text { messaging-twice-weekly } \\
\text { voice messages over } \\
14 \text { months promoting } \\
\text { behavior change to reduce } \\
\text { diabetes risk }\end{array}$ & $\begin{array}{l}\text { Mhealth-14 months. } \\
\text { Community } \\
\text { mobilization } \\
18 \text { months }\end{array}$ & $\begin{array}{l}\text { Lay facilitators for } \\
\text { community } \\
\text { mobilization. Mobile } \\
\text { phones for voice } \\
\text { messages }\end{array}$ & Community & $\begin{array}{l}18 \text { months } \\
\text { and } 2 \text { years }\end{array}$ & Usual care \\
\hline $\begin{array}{l}\text { Nguyen, } \\
2012[41]\end{array}$ & $\begin{array}{l}\text { Vietnam } \\
\text { (lower MIC) }\end{array}$ & $\begin{array}{l}\text { Controlled } \\
\text { before and } \\
\text { affer }\end{array}$ & $\begin{array}{l}\text { To evaluate the } \\
\text { impact of heatthy } \\
\text { lifestyle promotion } \\
\text { campaigns on } \\
\text { CVD risk factors in } \\
\text { the general } \\
\text { population in the } \\
\text { context of a } \\
\text { community-based } \\
\text { program on } \\
\text { hypertension } \\
\text { management }\end{array}$ & Rural population & $\begin{array}{l}\text { Community } \\
\text { inhabitants }\end{array}$ & $\begin{array}{l}25 \text { years } \\
\text { and above }\end{array}$ & 4,650 & $\begin{array}{l}\text { A hypertensive-targeted } \\
\text { management program } \\
\text { (monthly BP check, training } \\
\text { health workers and multidrug } \\
\text { therapy) integrated with a } \\
\text { community-targeted health } \\
\text { promotion on smoking } \\
\text { cessation, reducing alcohol } \\
\text { consumption, encouraging } \\
\text { physical activity and reducing } \\
\text { salty diets (healthy individuals) } \\
\text { delivered through lifestyle } \\
\text { promotion campaign through } \\
\text { broadcasting, leaflets or } \\
\text { meetings }\end{array}$ & 3 years & $\begin{array}{l}\text { Health workers, } \\
\text { mass media }\end{array}$ & Community & 3 years & $\begin{array}{l}\text { Routine } \\
\text { conventional } \\
\text { heathcare } \\
\text { services }\end{array}$ \\
\hline $\begin{array}{l}\text { Chandraratne, } \\
2019[42]\end{array}$ & $\begin{array}{l}\text { Sri Lanka } \\
\text { (lower MIC) }\end{array}$ & $\begin{array}{l}\text { Cluster } \\
\text { randomized } \\
\text { trial }\end{array}$ & $\begin{array}{l}\text { To investigate the } \\
\text { effect of an } \\
\text { intervention with } \\
\text { youth on } \\
\text { cardiovascular } \\
\text { disease risk }\end{array}$ & A semi-urban area & $\begin{array}{l}\text { Adults within } \\
\text { selected } \\
\text { divisions }\end{array}$ & $30-59$ years & 512 & $\begin{array}{l}\text { Youths as change agents } \\
\text { taking body weight and blood } \\
\text { pressure measurements and } \\
\text { proposed healthy lifestyle } \\
\text { behaviors to adults }\end{array}$ & 12 months & $\begin{array}{l}\text { Trained youths who } \\
\text { are members of a } \\
\text { club }\end{array}$ & Community & 12 months & No intervention \\
\hline
\end{tabular}


TABLE 1 | (Continued) Characteristics of included studies and community-based interventions, Community-based interventions for cardiovascular disease prevention in low- and middle-income countries: a systematic review, 2000-2019.

\begin{tabular}{|c|c|c|c|c|c|c|c|c|c|c|c|c|c|}
\hline Author & $\begin{array}{l}\text { Country } \\
\text { (income } \\
\text { category) }\end{array}$ & $\begin{array}{l}\text { Study } \\
\text { design }\end{array}$ & $\begin{array}{l}\text { Study } \\
\text { aim }\end{array}$ & Context & $\begin{array}{c}\text { Target } \\
\text { population }\end{array}$ & Age & Participants & Intervention & Length & Provider & Setting & $\begin{array}{l}\text { Follow-up } \\
\text { time }\end{array}$ & Control \\
\hline Joshi, 2019 [43] & $\begin{array}{l}\text { India } \\
\text { (lower MIC) }\end{array}$ & $\begin{array}{l}\text { Cluster } \\
\text { randomized } \\
\text { trial }\end{array}$ & $\begin{array}{l}\text { To assess impact } \\
\text { of CHW based } \\
\text { interventions in } \\
\text { reducing CVD risk } \\
\text { factors in rural } \\
\text { households in } \\
\text { India }\end{array}$ & Rural regions in India & $\begin{array}{l}\text { All households } \\
\text { with individuals } \\
\text { of age } \\
\geq 35 \text { years living } \\
\text { in these villages }\end{array}$ & $\begin{array}{l}\text { Individuals } \\
\text { aged } \\
35 \text { years } \\
\text { and above }\end{array}$ & $\begin{array}{l}\text { Individuals } \\
\text { resident } \\
\text { within } \\
\text { selected } \\
\text { villages }\end{array}$ & $\begin{array}{l}\text { CHWs delivered risk-reduction } \\
\text { advice and monitored risk } \\
\text { factors during } 6 \text { household } \\
\text { visits. CHWs measured blood } \\
\text { pressure, ascertained and } \\
\text { reinforced adherence to } \\
\text { prescribed therapies. The } \\
\text { CHWs also placed short goal- } \\
\text { directed slogans printed on } \\
\text { common household objects in } \\
\text { the household to promote } \\
\text { integration of preventive } \\
\text { therapies with activities of daily } \\
\text { living }\end{array}$ & 12 months & $\begin{array}{l}\text { Community health } \\
\text { workers }\end{array}$ & Community & $\begin{array}{l}12 \text { and } \\
18 \text { months }\end{array}$ & $\begin{array}{l}\text { Did not receive } \\
\text { CHW visits but } \\
\text { had access to a } \\
\text { clinic which was } \\
\text { run in the sub- } \\
\text { center location }\end{array}$ \\
\hline $\begin{array}{l}\text { Gunawardena, } \\
2016 \text { [44] }\end{array}$ & $\begin{array}{l}\text { Sri Lanka } \\
\text { (lower MIC) }\end{array}$ & $\begin{array}{l}\text { Cluster } \\
\text { randomized } \\
\text { controlled trial }\end{array}$ & $\begin{array}{l}\text { To examine the } \\
\text { effect of the child- } \\
\text { initiated } \\
\text { intervention on } \\
\text { weight, physical } \\
\text { activity and dietary } \\
\text { habit of their } \\
\text { mothers }\end{array}$ & $\begin{array}{l}\text { Semi-urban area of } \\
\text { colombo }\end{array}$ & $\begin{array}{l}\text { Sri lankan } \\
\text { mothers with a } \\
\text { school-aged } \\
\text { child }\end{array}$ & $\begin{array}{l}\text { Mean age } \\
\text { between } \\
37.5 \text { and } \\
38.5 \text { years }\end{array}$ & 308 & $\begin{array}{l}\text { Trained health promotion } \\
\text { facilitators visited the } \\
\text { intervention schools and } \\
\text { delivered the intervention in the } \\
\text { form of a series of discussion } \\
\text { on health and well-being with } \\
\text { the selected students with } \\
\text { emphasis on their mothers }\end{array}$ & 12 months & Students & Community & 12 months & No intervention \\
\hline Joshi, 2012 [45] & $\begin{array}{l}\text { India } \\
\text { (lower MIC) }\end{array}$ & $\begin{array}{l}\text { Cluster } \\
\text { randomized } \\
\text { controlled trial }\end{array}$ & $\begin{array}{l}\text { To develop, } \\
\text { implement, and } \\
\text { evaluate } 2 \text { CVD } \\
\text { prevention } \\
\text { strategies that } \\
\text { could. Potentially } \\
\text { be delivered by } \\
\text { NPHWs: } 1 \text { based } \\
\text { on a clinical. } \\
\text { Approach and } 1 \\
\text { based on health } \\
\text { promotion }\end{array}$ & $\begin{array}{l}\text { Rural villages in the east } \\
\text { and west godavari } \\
\text { districts of Andhra } \\
\text { Pradesh in India }\end{array}$ & $\begin{array}{l}\text { Residents of the } \\
\text { study area }\end{array}$ & $\begin{array}{l}30 \text { years or } \\
\text { older }\end{array}$ & 3,711 & $\begin{array}{l}\text { The campaign included } \\
\text { posters, street theater, rallies, } \\
\text { and community presentations } \\
\text { designed to convey messages } \\
\text { about stopping tobacco use, } \\
\text { heart-healthy eating, and } \\
\text { physical activity }\end{array}$ & 18 months & $\begin{array}{l}\text { Posters, street } \\
\text { theater, rallies }\end{array}$ & Community & 18 months & $\begin{array}{l}\text { In the villages } \\
\text { assigned to the } \\
\text { control group, } \\
\text { there was no } \\
\text { additional health } \\
\text { promotion } \\
\text { campaign } \\
\text { planned }\end{array}$ \\
\hline Lu, 2015 [46] & $\begin{array}{l}\text { China } \\
\text { (upper MIC) }\end{array}$ & $\begin{array}{l}\text { Randomized } \\
\text { controlled trial }\end{array}$ & $\begin{array}{l}\text { To evaluate } \\
\text { community-based } \\
\text { health education } \\
\text { strategies in the } \\
\text { management of } \\
\text { hypertensive } \\
\text { patients with low } \\
\text { socioeconomic } \\
\text { status in } \\
\text { dongguan city, } \\
\text { China }\end{array}$ & $\begin{array}{l}\text { Community health } \\
\text { service center in liabout } \\
\text { town in China }\end{array}$ & $\begin{array}{l}\text { Hypertensive } \\
\text { patients } \\
\text { managed at a } \\
\text { community } \\
\text { health service } \\
\text { center }\end{array}$ & $40-75$ years & 360 & $\begin{array}{l}\text { Three health education } \\
\text { groups. Self-learning reading } \\
\text { group - orientation on reading } \\
\text { materials to learn about } \\
\text { hypertension through posters } \\
\text { and booklets. Monthly regular } \\
\text { didactic lecture group by } \\
\text { phone invitation with lecture } \\
\text { lasting about } 30 \text { min. Monthly } \\
\text { interactive education } \\
\text { workshop-active involvement } \\
\text { of participants in use of visual } \\
\text { health education tools }\end{array}$ & 2 years & $\begin{array}{l}\text { Health education } \\
\text { materials developed } \\
\text { by CVD experts }\end{array}$ & Community & 2 years & $\begin{array}{l}\text { Groups } \\
\text { controlled for } \\
\text { each other }\end{array}$ \\
\hline
\end{tabular}


TABLE 1 ( (Continued) Characteristics of included studies and community-based interventions, Community-based interventions for cardiovascular disease prevention in low- and middle-income countries: a systematic review, 2000-2019.

\begin{tabular}{|c|c|c|c|c|c|c|c|c|c|c|c|c|c|}
\hline Author & $\begin{array}{l}\text { Country } \\
\text { (income } \\
\text { category) }\end{array}$ & $\begin{array}{l}\text { Study } \\
\text { design }\end{array}$ & $\begin{array}{l}\text { Study } \\
\text { aim }\end{array}$ & Context & $\begin{array}{c}\text { Target } \\
\text { population }\end{array}$ & Age & Participants & Intervention & Length & Provider & Setting & $\begin{array}{l}\text { Follow-up } \\
\text { time }\end{array}$ & Control \\
\hline Lv, 2014 [47] & $\begin{array}{l}\text { China } \\
\text { (upper MIC }\end{array}$ & $\begin{array}{l}\text { Controlled } \\
\text { before and } \\
\text { after }\end{array}$ & $\begin{array}{l}\text { To assess the } \\
\text { short-term impact } \\
\text { of a } \\
\text { comprehensive, } \\
\text { community-based } \\
\text { multilevel } \\
\text { intervention on } \\
\text { knowledge, beliefs } \\
\text { and practices with } \\
\text { respect to } \\
\text { smoking, physical } \\
\text { activity and diet in } \\
\text { hangzhou, China }\end{array}$ & $\begin{array}{l}\text { Three adjacent districts } \\
\text { located in a central } \\
\text { geographical location } \\
\text { of hangzhou were } \\
\text { included }\end{array}$ & $\begin{array}{l}\text { Individuals who } \\
\text { had lived in the } \\
\text { local district for } \\
\text { at least } 1 \text { year }\end{array}$ & 18-64 years & 2016 & $\begin{array}{l}\text { Community mobilization, } \\
\text { structural change in individual, } \\
\text { social, physical and policy } \\
\text { environment, health education } \\
\text { and social marketing }\end{array}$ & 2 years & $\begin{array}{l}\text { Staff of local } \\
\text { organization, heatth } \\
\text { workers, } \\
\text { community public } \\
\text { health assistants }\end{array}$ & $\begin{array}{l}\text { Neighboorhoods, } \\
\text { schools, } \\
\text { workplaces and } \\
\text { community health } \\
\text { center }\end{array}$ & 2 years & $\begin{array}{l}\text { Routine NCD } \\
\text { prevention and } \\
\text { control practices }\end{array}$ \\
\hline Chao, 2012 [48] & $\begin{array}{l}\text { China } \\
\text { (upper MIC) }\end{array}$ & $\begin{array}{l}\text { Randomized } \\
\text { controlled trial }\end{array}$ & $\begin{array}{l}\text { To evaluate the } \\
\text { impact of } \\
\text { community-based } \\
\text { health } \\
\text { management on } \\
\text { the health of the } \\
\text { elderly through an } \\
\text { RCT in naning, } \\
\text { China }\end{array}$ & $\begin{array}{l}\text { Naning in southeastem } \\
\text { China is the provincial } \\
\text { capital Of jiangsu } \\
\text { province-one of the } \\
\text { most developed } \\
\text { provinces in China }\end{array}$ & $\begin{array}{l}\text { Elderly persons } \\
\text { resident in the } \\
\text { area }\end{array}$ & $\begin{array}{l}60 \text { years } \\
\text { and above }\end{array}$ & 2,400 & $\begin{array}{l}\text { Health management program } \\
\text { including 1) health record } \\
\text { establishment; 2) health } \\
\text { evaluation; and 3) heath } \\
\text { management, including diet } \\
\text { advice, psychological aspects } \\
\text { of heatth, a tailor-made } \\
\text { exercise program, education/ } \\
\text { skills training on heath self- } \\
\text { management, telephone } \\
\text { consultation, lectures on } \\
\text { health, and distribution of } \\
\text { health promoting materials }\end{array}$ & 18 months & $\begin{array}{l}\text { Specifically trained } \\
\text { community health } \\
\text { senvice center staff, } \\
\text { managers and } \\
\text { related researchers }\end{array}$ & $\begin{array}{l}\text { Community health } \\
\text { service center }\end{array}$ & 18 months & Usual care \\
\hline $\begin{array}{l}\text { Huang, } \\
2011 \text { [49] }\end{array}$ & $\begin{array}{l}\text { China } \\
\text { (upper MIC) }\end{array}$ & $\begin{array}{l}\text { Controlled } \\
\text { before and } \\
\text { after }\end{array}$ & $\begin{array}{l}\text { To evaluate the } \\
\text { effects of a } \\
\text { community } \\
\text { intervention } \\
\text { program, which } \\
\text { focused on } \\
\text { improving the } \\
\text { hypertension } \\
\text { knowledge, diets } \\
\text { and lifestyles in a } \\
\text { rural Chinese area }\end{array}$ & $\begin{array}{l}\text { Rural area with } \\
\text { highlands and } \\
\text { agriculture resources } \\
\text { (oranges and tea) }\end{array}$ & $\begin{array}{l}\text { Rural residents } \\
\text { from xiaoxita } \\
\text { and fenxiang } \\
\text { towns }\end{array}$ & $\begin{array}{l}35 \text { and } \\
\text { above years }\end{array}$ & 1,632 & $\begin{array}{l}\text { Training local staff on } \\
\text { guidelines for hypertensions } \\
\text { prevention and treatment, and } \\
\text { education of participants } \\
\text { through using hypertension } \\
\text { education and dietary and } \\
\text { lifestyle guidance. Provided } \\
\text { pamphlets with information on } \\
\text { dietary and lifestyle behaviors } \\
\text { and one salt spoon (for } 2 \text { g) to } \\
\text { households }\end{array}$ & 3 years & $\begin{array}{l}\text { Trained local } \\
\text { healthcare staff, } \\
\text { media, research } \\
\text { team and materials }\end{array}$ & Community & 3 years & $\begin{array}{l}\text { Access to } \\
\text { normal, standard } \\
\text { health care } \\
\text { during the study } \\
\text { period }\end{array}$ \\
\hline Zhang, 2018 [50] & $\begin{array}{l}\text { China } \\
\text { (upper MIC) }\end{array}$ & $\begin{array}{l}\text { Randomized } \\
\text { controlled trial }\end{array}$ & $\begin{array}{l}\text { To investigate the } \\
\text { effectiveness of } \\
\text { the older-centered } \\
\text { integrated health } \\
\text { management } \\
\text { model project for } \\
\text { multiple lifestyle } \\
\text { behaviors in the } \\
\text { elderly }\end{array}$ & $\begin{array}{l}\text { Community health } \\
\text { centers in nanjing, } \\
\text { China }\end{array}$ & $\begin{array}{l}\text { Elderly persons } \\
\text { who had lived in } \\
\text { the community } \\
\text { for atleast } \\
2 \text { years }\end{array}$ & $60-80$ years & 671 & $\begin{array}{l}\text { One hour health education } \\
\text { program every two months } \\
\text { covering several topics } \\
\text { including chronic disease risk } \\
\text { factors, psychosocial support } \\
\text { and lifestyle changes, and a } \\
\text { two year health management } \\
\text { program that provided them } \\
\text { with information, skills and } \\
\text { tools for self-management, } \\
\text { family and community } \\
\text { management }\end{array}$ & 2 years & $\begin{array}{l}\text { Community health } \\
\text { service center staff }\end{array}$ & $\begin{array}{l}\text { Hospital or } \\
\text { community center }\end{array}$ & 24 months & Usual care \\
\hline
\end{tabular}


TABLE 1 ( (Continued) Characteristics of included studies and community-based interventions, Community-based interventions for cardiovascular disease prevention in low- and middle-income countries: a systematic review, 2000-2019.

\begin{tabular}{|c|c|c|c|c|c|c|c|c|c|c|c|c|c|}
\hline Author & $\begin{array}{l}\text { Country } \\
\text { (income } \\
\text { category) }\end{array}$ & $\begin{array}{l}\text { Study } \\
\text { design }\end{array}$ & $\begin{array}{l}\text { Study } \\
\text { aim }\end{array}$ & Context & $\begin{array}{c}\text { Target } \\
\text { population }\end{array}$ & Age & Participants & Intervention & Length & Provider & Setting & $\begin{array}{l}\text { Follow-up } \\
\text { time }\end{array}$ & Control \\
\hline $\begin{array}{l}\text { Ibrahim, } \\
2016[51]\end{array}$ & $\begin{array}{l}\text { Malaysia } \\
\text { (upper MIC) }\end{array}$ & $\begin{array}{l}\text { Controlled } \\
\text { before and } \\
\text { after }\end{array}$ & $\begin{array}{l}\text { To determine the } \\
\text { effects of a } \\
\text { community-based } \\
\text { lifestyle } \\
\text { intervention } \\
\text { delivered to adults } \\
\text { with pre-diabetes } \\
\text { and their health- } \\
\text { related quality of } \\
\text { life as compared to } \\
\text { the usual care } \\
\text { group }\end{array}$ & $\begin{array}{l}\text { Sub-urban } \\
\text { communities in } \\
\text { seremban. Malaysia }\end{array}$ & $\begin{array}{l}\text { Participants with } \\
\text { prediabetes }\end{array}$ & $18-65$ years & 297 & $\begin{array}{l}\text { Twelve group-based sessions } \\
\text { of } 90 \text { min each and minimum } \\
\text { of two individual counseling } \\
\text { sessions with the dietician and } \\
\text { researcher to reinforce } \\
\text { behavioral change. Group } \\
\text { sessions (i.e., lecture, seminar, } \\
\text { group work or discussion). } \\
\text { Community volunteers trained } \\
\text { in a two-day training workshop } \\
\text { prior to the delivery of } \\
\text { intervention }\end{array}$ & 12 months & $\begin{array}{l}\text { Dietetician and } \\
\text { researcher }\end{array}$ & Community & 12 months & $\begin{array}{l}\text { Standard health } \\
\text { education from } \\
\text { primary care } \\
\text { providers in the } \\
\text { clinic and } \\
\text { pamphlets. And } \\
\text { booklets about } \\
\text { various health } \\
\text { topics }\end{array}$ \\
\hline $\begin{array}{l}\text { Mcalister, } \\
2000[52]\end{array}$ & $\begin{array}{l}\text { Russia } \\
\text { (upper MIC) }\end{array}$ & $\begin{array}{l}\text { Controlled } \\
\text { before and } \\
\text { after }\end{array}$ & $\begin{array}{l}\text { To test the } \\
\text { feasibility and } \\
\text { effectiveness of } \\
\text { smoking reduction } \\
\text { that have proven } \\
\text { to be useful in } \\
\text { Finnish karelia }\end{array}$ & $\begin{array}{l}\text { Impoverished area with } \\
\text { difficult economic } \\
\text { conditions, low socio- } \\
\text { economic status, } \\
\text { poorly funded health } \\
\text { system and high } \\
\text { mortality }\end{array}$ & $\begin{array}{l}\text { Daily smokers in } \\
\text { pitkäranta and } \\
\text { adjacent district } \\
\text { of suojäni }\end{array}$ & $25-64$ years & 378 & $\begin{array}{l}\text { A smoking cessation } \\
\text { campaign "quit and win" with } \\
\text { media publicity (role modeling) } \\
\text { and community support }\end{array}$ & 1 year & $\begin{array}{l}\text { Staff of the } \\
\text { pitkëranta central } \\
\text { hospital }\end{array}$ & Community & 12 months & No intervention \\
\hline Aung, 2019 [53] & $\begin{array}{l}\text { Thailand } \\
\text { (upper MIC) }\end{array}$ & $\begin{array}{l}\text { Randomized } \\
\text { controlled trial }\end{array}$ & $\begin{array}{l}\text { To compare levels } \\
\text { of smoking } \\
\text { cessation } \\
\text { between the } \\
\text { intervention new } \\
\text { service package } \\
\text { arm and the } \\
\text { control routine } \\
\text { service arm over a } \\
\text { six-month period } \\
\text { and also compare } \\
\text { the smokerlyzer- } \\
\text { confirmed } \\
\text { smoking cessation } \\
\text { rates }\end{array}$ & $\begin{array}{l}\text { Rural districts where } \\
\text { people often grow } \\
\text { tobacco in their } \\
\text { gardens and consume } \\
\text { home-made hand- } \\
\text { rolled cigarettes }\end{array}$ & $\begin{array}{l}\text { Patients with } \\
\text { hypertension } \\
\text { and diabetes in } \\
\text { northern } \\
\text { Thailand }\end{array}$ & $35-80$ years & 319 & $\begin{array}{l}\text { Regular patient motivation by } \\
\text { the same nurse over a } \\
3 \text { month period, a monthly } \\
\text { picO + smokerlyzer test for } \\
3 \text { months, continual } \\
\text { assistance from a trained } \\
\text { family member, using a } \\
\text { smoking-cessation- diary; and } \\
\text { optional nicotine replacement } \\
\text { chewing gum therapy }\end{array}$ & 6 months & $\begin{array}{l}\text { Health workers and } \\
\text { family member }\end{array}$ & $\begin{array}{l}\text { Starts at primary } \\
\text { care into } \\
\text { community }\end{array}$ & 12 months & $\begin{array}{l}\text { Routine service } \\
\text { comprising of } \\
\text { brief counseling } \\
\text { and casual } \\
\text { follow-up }\end{array}$ \\
\hline Latina, 2020 [54] & $\begin{array}{l}\text { Grenada } \\
\text { (upper MIC) }\end{array}$ & $\begin{array}{l}\text { Randomized } \\
\text { controlled trial }\end{array}$ & $\begin{array}{l}\text { To test the } \\
\text { effectiveness of } \\
\text { peer support } \\
\text { strategy for } \\
\text { cardiovascular risk } \\
\text { reduction }\end{array}$ & $\begin{array}{l}\text { Residents were from } \\
\text { five parishes around } \\
\text { the island }\end{array}$ & $\begin{array}{l}\text { Adults from the } \\
\text { Grenada heart } \\
\text { project cohort } \\
\text { study of age with } \\
\text { at least two } \mathrm{CV} \\
\text { risk factors }\end{array}$ & $18-85$ years & 402 & $\begin{array}{l}\text { CHWs were trained to deliver } \\
\text { the community-based } \\
\text { program over a } 5 \text { days course. } \\
\text { The intervention group was } \\
\text { organized into groups of 8-12 } \\
\text { individuals in their local parish. } \\
\text { A "peer leader" underwent } \\
\text { additional training in leadership } \\
\text { and communication and heath } \\
\text { behavior communication }\end{array}$ & 12 months & Peer leaders & Community & 12 months & $\begin{array}{l}\text { Series of } \\
\text { educational } \\
\text { lectures at the } \\
\text { time of } \\
\text { enrollment, } \\
\text { followed by self- } \\
\text { management for } \\
1 \text { year }\end{array}$ \\
\hline $\begin{array}{l}\text { Anthony, } \\
2015[55]\end{array}$ & $\begin{array}{l}\text { China } \\
\text { (UpperMIC), } \\
\text { India (lower } \\
\text { MIC), Mexico } \\
\text { (upperMIC) }\end{array}$ & $\begin{array}{l}\text { Controlled } \\
\text { before and } \\
\text { after }\end{array}$ & $\begin{array}{l}\text { To reduce risk } \\
\text { factors in } \\
\text { workplace settings } \\
\text { in low- and } \\
\text { middle-income } \\
\text { countries }\end{array}$ & $\begin{array}{l}\text { Intervention and } \\
\text { control areas in each } \\
\text { country had similar } \\
\text { demographic. And } \\
\text { socioeconomic } \\
\text { characteristics with a } \\
\text { population size. } \\
\text { Between } 150,000 \text { and } \\
200,000\end{array}$ & $\begin{array}{l}\text { All workers aged } \\
18-64 \text { years in } \\
\text { each workplace }\end{array}$ & $18-64$ years & 12,136 & $\begin{array}{l}\text { Heatth education, structural } \\
\text { change though policies, } \\
\text { mandates and restrictions, and } \\
\text { community mobilization, } \\
\text { including no smoking days and } \\
\text { smoking bans }\end{array}$ & 18-24 months & $\begin{array}{l}\text { Community } \\
\text { coallitins consisting } \\
\text { of key decision } \\
\text { makers, media }\end{array}$ & $\begin{array}{l}\text { Workplaces, } \\
\text { schools, and } \\
\text { community }\end{array}$ & $\begin{array}{l}18 \text { and } \\
24 \text { months }\end{array}$ & No intervention \\
\hline
\end{tabular}




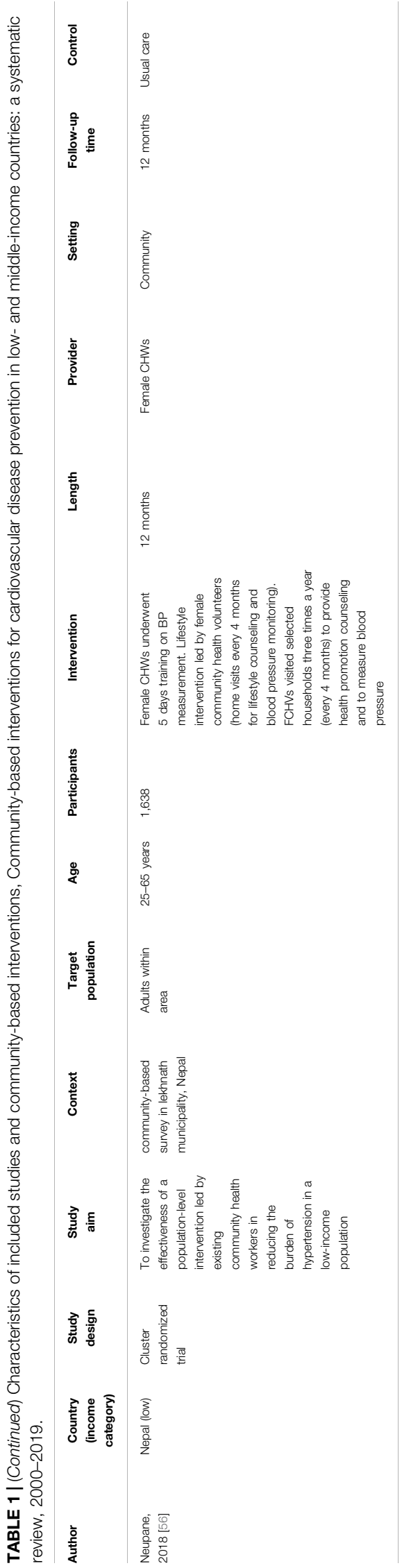

and control groups and eight with low (1), moderate/some concerns (7) RoB found changes in favor of the intervention group in engaging in physical activity [26, 41, 44], leisure-time activity [44], proportion of participants physically active [30, 32, 51], adherence to physical activity [50] and energy expenditure for physical activities [26, 39]. In one study with a serious RoB, significant improvements were higher in the control group [49] while there was no significant difference between groups in the remaining studies all of which were of low, moderate or some concerns RoB [34, 37, 42, 43, 54-56]. In seven studies, differences in physical activity were tested within groups and significant improvements were noted in the intervention group [26, 27, 47, 48], both intervention and control groups [46] or none of them $[36,38,40]$. Some studies compared physical activity levels between males and females and found more activity among males than females [41] and higher activity levels among females compared to males [26]. Majority of effective studies utilized health education through mass media, public places, and information, education and communication materials, training of health workers and community volunteers, community mobilization through campaigns and structural changes in the environment.

\section{Diet}

Twenty-one studies determined changes in diet following implementation of interventions. Fruits and vegetable consumption significantly improved among intervention compared to control group in five studies of low [34], some concerns [40], moderate [26, 55], or high RoB [38] unlike others $[32,33,44,45,56]$. In one study with some concerns RoB, the changes were only observed in fruit intake [42]. Several studies also showed significant differences in favor of the intervention in lower salt consumption [41, 49, 55], fat intake [26, 45, 49] and snacks consumption [42] when compared with control groups. Other studies measured overall diet quality and found significantly greater improvements in diet score or adherence to recommended diets in intervention populations relative to control group $[37,48,50]$. In six studies, there were no significant differences between the intervention and control groups in dietary components examined [30, 36, 46, 47, 51, 56]. Effective dietary interventions mostly focused on providing advice on healthy diets, community health education during meetings and public places, community mobilization, tailoring interventions based on readiness to change and follow up of persons such as through phone calls.

\section{Alcohol Use}

Eight studies explored outcomes related to alcohol consumption as a risk factor for CVD [32, 34, 41, 42, 46, 49, 50, 56]. In two studies of low and serious RoB, intervention group participants exhibited a greater reduction in alcohol consumption compared to control participants [34, 49] while in another study of moderate RoB, significant differences were observed in both intervention and control communities [41]. Effective interventions for alcohol reduction were mainly communitybased peer support with group sessions, community-wide activities such in public places, health education through 
pamphlets, newspapers, classes and training of health workers. In the remaining studies with some concerns RoB, there was no significant reduction in proportion of persons consuming alcohol $[42,46,56]$ or adhering to its moderate use [50]. On the other hand, significant reduction in alcohol use at population level and among patients with hypertension was observed in the control group of a study with moderate RoB conducted in Kenya [32].

\section{Smoking}

Among the 19 studies that examined changes in smoking levels, nine with low (2), moderate/some concerns (4), high/serious (3) RoB found a statistically significant difference in smoking prevalence in favor of the intervention compared to the control group [26, 35, 38, 39, 43, 47, 52, 53, 55]. Indeed, these differences were in smoking cessation [26, 35, 39, 47, 52, 53, 55] and reduced use of smokeless tobacco [38, 43]. In two controlled before and after studies with moderate RoB, stratified analysis found significantly greater reductions in smoking cessation among men but not women [26, 55]. Effective smoking interventions involved health education through social gatherings, pamphlets, brochures and booklets; community mobilization through training, involvement of local leaders and use of media campaigns; and group or individual counseling. In ten studies with low (3), moderate (2), some concerns (3), high (1) and serious (1) RoB, there was no statistically significant difference in smoking measures between the intervention and control groups [32, 34, 41, 42, 45, 46, 49, 50, 54, 56].

\section{Secondary Outcomes Body Weight}

Fourteen studies assessed changes in body weight among study participants with six of low (2), moderate (1) and some concerns (3) RoB reporting significant reduction in body mass index [42, 44, 46], waist hip ratio [48], body weight [30, 36, 42, 44] and abdominal obesity [30] in intervention compared to control groups. Eight studies of low (3), some concerns/moderate (4) and high (1) RoB did not find any significant difference between groups [34, 37, 39, 41, 45, 50, 51, 55]. Health education groups, health management programmes including training and counseling, nutrition education classes, training and engaging community volunteers were effective interventions for reducing body weight indicators.

\section{Blood Pressure}

Following implementation of interventions among the population groups, compared with the control groups, in seven of the 16 studies with low (1) and some concerns/ moderate (6) RoB, the intervention groups registered significant reductions in systolic [39, 48, 56] and diastolic blood pressure [30,51] or both [41, 50]. In addition, two studies with high/serious RoB demonstrated a positive effect of the intervention on awareness, treatment and control of hypertension [49] and adherence to antihypertensive drugs [43]. Studies that showed effectiveness in blood pressure outcomes involved several strategies including health education awareness raising through mass media and public places, trainings, lifestyle promotion campaigns, health management programmes providing information, skills and tools for self-management, counseling sessions and improving access to blood pressure screening. There was no significant difference observed between intervention and control groups in systolic $[32,43,51]$ and diastolic $[39,48]$ blood pressure or both [34, 37, 40, 42, 45], and hypertension prevalence [30, 32, 40] and control [39, 40,46] even though within group differences were observed in some of the studies.

\section{Blood Glucose}

The ten studies that evaluated blood glucose related outcomes with low (4) and some concerns/moderate (6) RoB found significant reductions in fasting blood sugar [30, 48, 50, 51, 54], and prevalence of diabetes and intermediate hyperglycaemia [40], risk of developing diabetes [34] and incidence of diabetes $[26,36,37]$ in the intervention compared to control group. In one study with a low RoB, there was no significant difference in the incidence of diabetes between the intervention and control groups [34]. In another study with some concerns RoB, although differences were observed in the community mobilization arm, the m-health intervention arm did not influence the combined prevalence of intermediate hyperglycaemia and diabetes or the incidence of diabetes [40]. Interventions among effective studies included health education and advice, trainings, nutrition education through classes and print media such as brochures and pamphlets, provision of information and tools for self-management, secondary prevention activities in clinics and counseling.

\section{Lipids}

Seven studies evaluated changes in blood lipids and in four studies with low (1) and some concerns/moderate (3) RoB, significantly lower measures were registered for low density lipoprotein (LDL) [30, 37], triglycerides [30, 46] and total cholesterol $[30,46,50]$ and a high prevalence of high density lipoprotein (HDL) [51] in intervention compared to control groups. Conversely, within the intervention group of a study in Iran with moderate RoB, prevalence of low HDL cholesterol increased at follow-up [30]. Health education sometimes personalized for individuals to support lifestyle changes or conducted in groups through classes and print media or mobile messages, health management programmes, and secondary prevention activities in clinics formed effective interventions. There was no statistically significant difference between intervention and control groups in total cholesterol $[34,46]$, LDL cholesterol [34, 46], serum cholesterol [37] and triglycerides $[34,37,48]$ in other studies.

\section{DISCUSSION}

This systematic review examined community-based interventions for CVD prevention in LMICs and their effectiveness. Among the 27 studies that were included in the review, most employed health education and awareness creation, trainings, lifestyle consultation and counseling and community 
mobilization. Community-based interventions successfully improved population knowledge on CVD and risk factors and influenced physical activity and dietary practices unlike reduction in smoking and alcohol consumption. The interventions also led to significant improvements in blood pressure and blood sugar measurements. Overall, the effective interventions mostly involved community mobilization and social activities; health education and communication through use of information, education and communication materials including mass media; individual or group counseling; and trainings of providers including community health workers, peers or health workers.

Health education and health communication includes verbal and written measures to influence and empower individuals, populations, and communities to make healthier choices [58]. Many studies included more than one intervention strategy similar to other reviews [59] which is desirable as multistrategy interventions have been demonstrated to be more effective in influencing uptake of behaviors compared to individual strategies [58]. These strategies aim at changing people's knowledge, attitudes and/or behaviors and are considered a crucial first step in addition to community mobilization activities that help create a favourable enabling environment [58]. In addition, with the huge knowledge and capacity gaps in CVD services delivery in many LMICs [60-62], the high number of training interventions is not surprising.

The review also examined the effectiveness of communitybased interventions in increasing knowledge and uptake of CVD prevention practices. We found significant improvements in knowledge on behavioral and metabolic risk factors for CVD prevention in favor of the community-based interventions similar to a previous review [14]. These improvements were achieved mostly through training, community mobilization, health education and consultation [38, 40,45, 46, 49]. Worthy of note is that knowledge on CVD is still very low in many LMICs $[63,64]$ and thus interventions are more likely to lead to significant changes. Regarding, changes in behavioral practices following implementation of intervention, greatest improvements were around physical activity with increase in proportion physically active and leisure-time activity. Also, improvements in dietary practices were observed with changes reported in fruit and vegetable consumption, salt intake, and fats and snacks intake. An earlier review also observed improvements in physical activity and diet as significant lifestyle changes reported by a sizable proportion of studies [19]. Improvements in other risk factors were inconsistent with only few studies reporting changes in smoking and alcohol consumption behaviors. In high-income contexts, changes in smoking have been reported following community programmes [59]. Changes in alcohol and smoking practices usually takes a longer time yet many studies in our review had shorter follow-up periods of one to two years. Moreover, sustaining changes in behavior over long periods has been shown to be challenging [65, 66]. In some studies, the intensity of the intervention was low with broad interventions and self-reported outcome measures which gaps should be bridged in future studies. Moreover, there is need for further studies of longer durations to examine changes in CVD prevention practices and inform the growing evidence base for CVD prevention intervention effectiveness in LMICs. This is especially important because lifestyle interventions have been reported to be more cost effective than pharmacological interventions [67] which most LMICs cannot afford amidst the growing epidemic of NCDs [13]. When planning such intervention studies, multiple intervention strategies should be considered, adapted to context and informed by appropriate theories, frameworks and models and evaluated using robust study designs.

This review also examined changes in metabolic risk factors as secondary outcomes and found more evidence in favor of reduction in systolic blood pressure, diastolic blood pressure and blood glucose following community-based interventions which was from relatively lower ROB studies. Effectiveness of community-based intervention in reducing metabolic risk factors has been reported previously for several risk factors including systolic blood pressure $[14,19,68,69]$, diastolic blood pressure [69], incidence of diabetes [70] and HbA1C [70] including in high-income countries. Positive changes in behavioral risk factors should lead to improvements in metabolic risk factors for example changes in dietary behaviors and/or physical activity should impact blood pressure indicators. Although changes in metabolic risk factors were examined for only included studies as secondary outcomes and thus not comprehensive, this review provides insights into how community-based interventions impact both primary and secondary level CVD risk factors.

Overall, this systematic review notes that there have been interventions for CVD prevention in LMICs but these are mostly limited to middle-income Asian countries which could have been influenced by their higher CVD burden. The lack of studies from low-middle income sub-Saharan African countries is concerning and calls for systematic approaches to address the gaps. Targeted research funding and establishing dedicated research centers to support evidence generation and translation is recommended. Although majority of the studies were randomized controlled (17), only seven had a low risk of bias which affects the strength of currently available evidence. The design of future studies should consider measures to minimize identified sources of bias where possible including using objective measures of outcomes and/or using validated tools, properly designing interventions to avoid foreseeable deviations and applying appropriate techniques to deal with missing data. Moreover, randomized studies should ensure that randomization is effectively done to minimize baseline imbalances in study characteristics while non-randomized studies should control for most sources of confounding. We note that although the review intended to provide a comprehensive overview of community-based interventions and literature search widely done, the strict inclusion criteria designed to provide robust evidence on intervention effectiveness could have limited this. This review only considered studies published in the English language due to inadequate resources which could have led to publication bias hence future reviews should consider including other languages. We also excluded conference abstracts which 
TABLE 2 | Effect of community-based interventions on study outcomes, Community-based interventions for cardiovascular disease prevention in low- and middle-income countries: a systematic review, 2000-2019.

\begin{tabular}{|c|c|c|c|c|c|c|c|c|c|}
\hline Author & Knowledge & $\begin{array}{l}\text { Physical } \\
\text { activity }\end{array}$ & Diet & Smoking & Alcohol & Body weight & Blood pressure & Blood glucose & Lipid profile \\
\hline $\begin{array}{l}\text { Baghaei, } \\
2010 \text { [25] }\end{array}$ & & $\begin{array}{l}\text { Regular dalily exercise } \\
\text { significantly increased by } \\
\text { about } 45 \% \text { among the high } \\
\text { risk population in the } \\
\text { interventional area (from } 15 \\
\text { to } 28 \% \text { ) }\end{array}$ & $\begin{array}{l}\text { Improved fruit and vegetable } \\
\text { consumption, more ese of } \\
\text { unsaturated fats and reduced } \\
\text { salt intake among the high } \\
\text { risk population in the } \\
\text { intervention group compared } \\
\text { to reference group }(p<0.05)\end{array}$ & $\begin{array}{l}\text { Smoking significantly } \\
\text { decreased among the high } \\
\text { risk persons in the } \\
\text { intervention nroup compared } \\
\text { to reference group }(0<0.03)\end{array}$ & & & & & \\
\hline $\begin{array}{l}\text { Kelishadi, } \\
2011 \text { [26] }\end{array}$ & & $\begin{array}{l}\text { Leisure time physical activity } \\
\text { increased in women and } \\
\text { declined in men of both } \\
\text { communities with changes } \\
\text { greater in the intervention area } \\
\text { No significant change in } \\
\text { transport physical activity in } \\
\text { intervention area but sharply } \\
\text { decreased in reference area } \\
\text { Regular morning curricular } \\
\text { exercise significicantly was } \\
\text { greater in the intervention } \\
\text { community compared to } \\
\text { control i }(0<0.001)\end{array}$ & 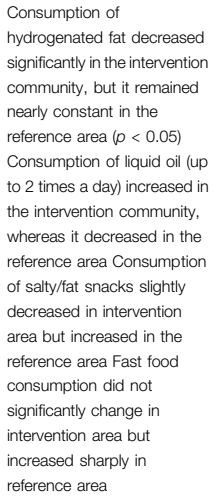 & $\begin{array}{l}\text { Prevalence of current } \\
\text { smoking decreased in men } \\
\text { living in initervention area but } \\
\text { increased slightly among } \\
\text { those in reference area. } \\
\text { However, no significant } \\
\text { change was observed } \\
\text { among women in both } \\
\text { groups Attempt to smoking } \\
\text { decreased among youths in } \\
\text { intervention areas but } \\
\text { remained constant in the } \\
\text { reference area }\end{array}$ & & & & & \\
\hline Rabiei, 2010 [27] & & $\begin{array}{l}\text { From } 2000 \text { to } 2002 \text {, the daily } \\
\text { physical activity (PA) among } \\
\text { both genders decreased in } \\
\text { both intervention and } \\
\text { reference communities } \\
\text { Leisure-time PA increased } \\
\text { significantly in the intervention } \\
\text { area, but decreased in the } \\
\text { reference area The } \\
\text { transportation PA did not } \\
\text { significantly change in the } \\
\text { intervention area, but showed } \\
\text { a remarkable decline in the } \\
\text { reference area among both } \\
\text { genders. No significant } \\
\text { change in worksite PA. }\end{array}$ & & & & & & & \\
\hline $\begin{array}{l}\text { Sarrafzadegan, } \\
2009 \text { [28] }\end{array}$ & & $\begin{array}{l}\text { Energy expenditure for total } \\
\text { daily physical activities } \\
\text { showed a decreasing trend in } \\
\text { all areas, but the mean drop } \\
\text { from baseline was significantly } \\
\text { smaller in the intervention } \\
\text { areas than in the control area } \\
\text { (-68 metabolic equivalent task } \\
\text { (MET) minutes per week vs. } \\
-114 \text { MET minutes per week, } \\
\text { respectively; } p<0.05) \text {. } \\
\text { Leisure time devoted to } \\
\text { physical activities showed an } \\
\text { increasing trend in all areas }\end{array}$ & $\begin{array}{l}\text { Changes from baseline in } \\
\text { mean dietary score differed } \\
\text { significantly between the } \\
\text { interention and control areas } \\
(+2.1 \text { points vs. }-1.2 \text { points, } \\
\text { respectively; } p<0.01) \text {, as did } \\
\text { the change in the percentage } \\
\text { of individuals following a } \\
\text { healthy diet ( }+14.9 \% \text { vs. } \\
-2.0 \% \text {, respectively; } p< \\
0.001)\end{array}$ & $\begin{array}{l}\text { Daily smoking had } \\
\text { decreased by } 0.9 \% \text { in the } \\
\text { intervention areas and by } \\
2.6 \% \text { in the control area at } \\
\text { the end of the third year, but } \\
\text { the difference was not } \\
\text { significant. Analysis by } \\
\text { gender revealed a significant } \\
\text { decreasing trend in smoking } \\
\text { among men }(p<0.05) \text { but } \\
\text { not among women }\end{array}$ & & & & & \\
\hline
\end{tabular}


TABLE 2 | (Continued) Effect of community-based interventions on study outcomes, Community-based interventions for cardiovascular disease prevention in low- and middle-income countries: a systematic review,

\begin{tabular}{|c|c|c|c|c|}
\hline Author & Knowledge & $\begin{array}{l}\text { Physical } \\
\text { activity }\end{array}$ & Diet & Smoking \\
\hline $\begin{array}{l}\text { Sarafzadegan, } \\
2009 \text { [29] }\end{array}$ & & $\begin{array}{l}\text { Intervention activities } \\
\text { positively affected the total } \\
\text { and leisure-time physical } \\
\text { activities in men, but not } \\
\text { women Total daily physical } \\
\text { activity decreased in both } \\
\text { groups over the evears but } \\
\text { trend was slower in } \\
\text { intervention group }(0<0.001)\end{array}$ & $\begin{array}{l}\text { Intervention resulted into } \\
\text { gradual improvement in the } \\
\text { nutrtitional status compared } \\
\text { to control with trends similar } \\
\text { in men and women }(p< \\
0.001) \text { Dietary choice of both } \\
\text { sexes showed modest } \\
\text { degrees of improvement }\end{array}$ & $\begin{array}{l}\text { Smoking status of men } \\
\text { improved during the study } \\
\text { period (except 2004), while } \\
\text { the effect on women was not } \\
\text { significant }\end{array}$ \\
\hline Azzizi, 2013 [30] & & $\begin{array}{l}\text { After intervention, chance for } \\
\text { being less active was } \\
\text { significantly higher in control } \\
\text { men as compared to } \\
\text { intervention men after } \\
3.6 \text { years: } \mathrm{OR}=1.2\end{array}$ & $\begin{array}{l}\text { No significant difference in } \\
\text { energy intake and } \\
\text { macronutrient consumption } \\
\text { between two groups at } \\
\text { baseline and after } \\
\text { intervention }\end{array}$ & \\
\hline
\end{tabular}

Ancolo

Body weight

Blood pressure

Blood glucose

Lipid profile

Mimiran,
$2008[3]]$

Mean dietary carbohydrate, mean dietary protein and fat
intakes decreased in both group but significant in intervention group. After adjustments, only total dietary cholesterol had a significant decrease $(p<0.05$. While the dietary vitamin a values decreased in controls, it Vitamin $B 6, B 12$, and $C$ and zinc intakes increased significantly in both gro while the iron intakes significantly decreased in bo groups. No significant difference in energy and nutrient intakes between Significant decrease in the inadequate physical activity among intervention compared control group at population level (OR $0.20, p<0.001)$

\begin{tabular}{|c|c|c|}
\hline 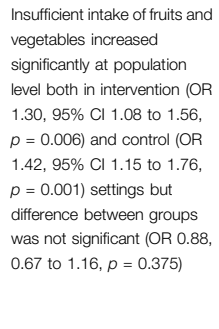 & $\begin{array}{l}\text { In the control group, there } \\
\text { was significant decrease at } \\
\text { population level in smoking } \\
\text { OR } 0.73,95 \% \mathrm{CI} \\
0.56-0.95) \text {. The difference in } \\
\text { intervention group was not } \\
\text { significant (OR } 1.19,95 \% \mathrm{Cl} \\
0.92 \text { to } 1.53, p=0.181) \text {. } \\
\text { Among patients with } \\
\text { hypertension in the control } \\
\text { group, smoking (OR } 0.51 \text {, } \\
95 \% \mathrm{Cl} 0.28 \text { to } 0.90, p= \\
0.021) \text { reduced significantly }\end{array}$ & $\begin{array}{l}\text { Significant reduction in } \\
\text { alcohol use at population } \\
\text { level in the control group (OR } \\
0.71,95 \% \text { Cl } 0.57-0.88) \text {. } \\
\text { Among patients with } \\
\text { hypertension in the control } \\
\text { group, alcohol use (OR } 0.62 \text {, } \\
95 \% \text { Cl } 0.38 \text { to } 0.99, p= \\
0.044) \text { reduced significantly }\end{array}$ \\
\hline
\end{tabular}

Prevalence of abdominal obesity increased significanty in both intervention and between groups revealed significant reduction in abdominal obesity in intervention group compared to control (OR 1.24, $1.07-1.44, p<0.014)$

Elevated blood pressure educed in both intervention

Ifference between groups w not statisitically significant

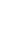

Diastollic blood pressure decreased significantty in bot groups but higher in the Systollic blood pressure increased significantly in the control group with no significant difference with
intervention group $(p=0.13)$ blood sugar in intervention group and significant increase between intervention and contro group was statisticaly significant $(p<0.01)$ cholesterol in the intervention
Prevalence of elevated fastin glucose increased only in the control group. On group group had significantly
reduced elevated fasting glucose (OR 1.67, CI 1.43-1. $195, p<0.001$ )

Prevalence of low HDL cholesterol increased
significantly in both groups while that of elevated baseline values. Compared to
control, interention significantly reduced ele triglycerides (OR 1.18, Cl $1.04-1.34, p<0.014)$ and low
HDL cholesterol (OR 1.52, C $1.32-1.76, p<0.001$ )

Total cholesterol and HDL and DL decreased significantly both groups. There was group compared to controls $(p=0.01)$
No significant reduction in mean SBP and DBP at population evel. No significant difference in SBP reauction among patients
with hypertension. However, DBP decreased more in the control group than the intervention one $(p=0.028)$. Significant reduction in SBP and DBP in both intervention $2.75 \mathrm{mmHg}(\rho=0.001)$ and $0.029)$ and larger reductions among those with hypertension in (intervention (SBP

$4.82 \mathrm{mmHg}, P<0.001 ; \mathrm{DB}$ $7.55 \mathrm{mmHg}, p<0.0011$ and control (SDP $14.05 \mathrm{mmHg}, P<$ $0.001 ;$ DBP $10.67 \mathrm{mmHg}, p<$ 
TABLE 2 | (Continued) Effect of community-based interventions on study outcomes, Community-based interventions for cardiovascular disease prevention in low- and middle-income countries: a systematic review, 2000-2019.

\begin{tabular}{|c|c|c|c|c|}
\hline Author & Knowledge & $\begin{array}{l}\text { Physical } \\
\text { activity }\end{array}$ & Diet & Smoking \\
\hline $\begin{array}{l}\text { Daivadanam, } \\
2018 \text { [33] }\end{array}$ & & & $\begin{array}{l}\text { Significant, modest increase } \\
\text { in fruit intake from baseline in } \\
\text { the intervention arm (12.5\%) } \\
\text { and control (6.6\%) but no } \\
\text { difference between the } \\
\text { groups. Significant increase } \\
\text { in vegetable intake in } \\
\text { intervention (13.99\%) and } \\
\text { control arms }(13.66 \%) \text { but no } \\
\text { difference between the } \\
\text { groups. Significant increase } \\
\text { in vegetable procurement by } \\
19 \% \text { in the intervention am } \\
\text { compared to the control arm } \\
(p=0.008) \text {. Monthly } \\
\text { housenold consumption of } \\
\text { salt, sugar and oil was greatly } \\
\text { reduced in the intervention } \\
\text { arm }(p<0.001)\end{array}$ & \\
\hline $\begin{array}{l}\text { Thankappan, } \\
2018[34]\end{array}$ & & $\begin{array}{l}\text { No statistically significant } \\
\text { difference in leisure time } \\
\text { physical activity between } \\
\text { intervention and control } \\
\text { groups }(\mathrm{RR}=1.20, p=0.36 \text { ) }\end{array}$ & $\begin{array}{l}\text { Intervention participants } \\
\text { more likely to consume } 5 \text { or } \\
\text { more servings of fruits and } \\
\text { vegetables (RR } 1.83, p= \\
0.008 \text { ) compared with control }\end{array}$ & $\begin{array}{l}\text { No statistically significant } \\
\text { difference in tobacco use } \\
\text { between intervention and } \\
\text { control groups (RR }=0.79 \text {, } \\
p=0.11)\end{array}$ \\
\hline
\end{tabular}

Body weight waist circumference (mean
Intervention participants had No statistically difference in a greater reduction alcohol use $($ RR $0.77, p=0.018)$
the amount of alcohol consuned was lower among intervention participants $(p=0.030)$ difference: $0.67, p=0.14$ ) an
waist to hip ratio (mean difference: $0.008, p=0.12$
between intervention and control groups
No statistically difference in systollic (mean difference: 1.22 ,
$P=0.13$ ) and diastollic blood $p=0.13$ ) and diastollic blo pressure (mean difference:
$1.12, p=0.06$ ) between intervention and control groups

Daboles developed in $17.1 \%$ (79/463) of control participa and $14.9 \%(68 / 456)$ of relative risk [RP] $0.88,95 \% \mathrm{Cl}$ $0.66 \pm 1.16, p=0.36$. group, intervention participants had a greater reduction in IDRS score (mean difference: -1.50 points, $p=$

Overall prevalence of smoking abstinence was $6.8 \%$ in the control group (relative risk: $1.85,95 \% \mathrm{Cl}$ 1.05, 3.25). A total of $41.3 \%$ subjects in the intervention area and $13.6 \%$ in the control area had reduced
smoking by $50 \%$ or more the end of 12 months $58.8 \%$ in LSM and from $45.9 \%$ to $62.9 \%$ in LSM + MET group adherence registered in LSM

\section{Significant increase in mean}

body weight in the control

group at annual follow-up.
Among intervention, increase

was only in the lifestyle group

at 24 months $(p=0.035)$

Mean waist circumference did

not significant differ in any

group relative to baseline
values

No significant effect of

intervention on BMl and waist

circumference

\section{No significant effect of} diastollic blood pressure
ditic
Significant relative risk

in the LSM $28.5 \%, p=0.018$ MET group $(p=0.029)$ and $28.2 \%$ in LSM + MET group $(p=0.022)$ compared to control group

$50(18 \%)$ of men in intervention group developed type 2 $(27 \%)$ in the control group (absolute risk reduction of 9 Intervention thus reduced incidence of type 2 diabetes. $(p=0.015)$

No significant effect of intervention on serum cholesterol and triglycerides cholesterol was significant (Continued on following page)
No statistically significant roups for total cholester (mean difference: 0.01, $p=$ 0.79), LDL cholesterol (mean triglycerides (mean difference $0.96, p=0.07$ ) 
TABLE 2 | (Continued) Effect of community-based interventions on study outcomes, Community-based interventions for cardiovascular disease prevention in low- and middle-income countries: a systematic review, 2000-2019.

\begin{tabular}{|c|c|c|c|c|c|c|c|c|c|}
\hline Author & Knowledge & $\begin{array}{l}\text { Physical } \\
\text { activity }\end{array}$ & Diet & Smoking & Alcohol & Body weight & Blood pressure & Blood glucose & Lipid profile \\
\hline Nishtar, 2007 [38] & 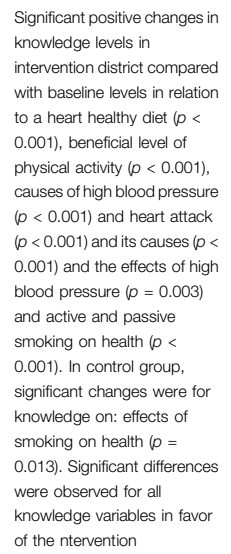 & $\begin{array}{l}\text { No changes were seen in the } \\
\text { level of physical activity }\end{array}$ & $\begin{array}{l}\text { Significant differences noted } \\
\text { in consumption of two or } \\
\text { more servings of vegetables } \\
\text { per day within the intervention } \\
\text { group }(p<0.001) \text { and } \\
\text { between the intervention and } \\
\text { control group at the end of } \\
\text { the intervention (0.020). No } \\
\text { significant differences } \\
\text { observed regarding } \\
\text { consumption of five or more } \\
\text { servings of fruits and } \\
\text { vegetables, consumption of } \\
\text { twwo or more fruit servings and } \\
\text { type of oilffat/ghee used for } \\
\text { cooking }\end{array}$ & $\begin{array}{l}\text { No significant changes } \\
\text { observed comparing pre and } \\
\text { post smoking pattern in both } \\
\text { intervention and control } \\
\text { groups. In comparing } \\
\text { intervention and control sites } \\
\text { post scores, there was a } \\
\text { difference in usage of } \\
\text { smokeless tobacco ( } p= \\
0.022)\end{array}$ & & & & & \\
\hline Jafar, 2009 [39] & & $\begin{array}{l}\text { Median metabolic equivalent } \\
\text { scores for physical activity } \\
\text { increased in the HHE and GP } \\
\text { group remained unchanged in } \\
\text { the GP only and HHE only } \\
\text { groups, and decreased in the } \\
\text { no intervention group ( } p= \\
0.030 \text { for difference among } \\
\text { groups) }\end{array}$ & & $\begin{array}{l}\text { Proportion of current } \\
\text { smokers decreased from } \\
\text { baseline to the last follow-up } \\
\text { visiti in all } 4 \text { groups }(p<0.001 \\
\text { in each). A } 12.3 \% \text { decrease } \\
\text { in the HHE and GP group, an } \\
11.9 \% \text { decrease in the HHE- } \\
\text { only and GP.-only groups, } \\
\text { and a } 9.5 \% \text { decrease in the } \\
\text { no intervention group }(p< \\
\text {..001 for difference among } \\
\text { groups) }\end{array}$ & & 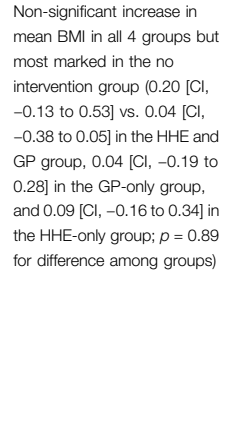 & 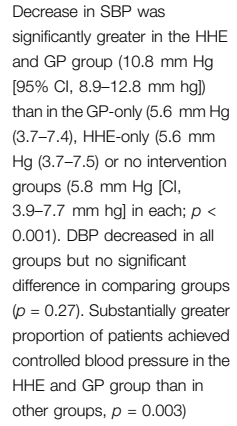 & & \\
\hline Fottrell, 2019 [40] & $\begin{array}{l}\text { Increases in ability to report } \\
\text { one or more valid causes, } \\
\text { symptoms, complications, } \\
\text { and strategies for prevention } \\
\text { and control of diabetes were } \\
\text { observed in both intervention } \\
\text { groups compared with } \\
\text { control, with the effect } \\
\text { consistently greatest in the } \\
\text { PLA group }(p<0.01)\end{array}$ & $\begin{array}{l}\text { No significant difference in } \\
\text { participants achieving an } \\
\text { average of more than } 150 \mathrm{~min} \\
\text { of physical activity per week } \\
\text { among the intervention } \\
\text { groups (PLA } 0.83(0.53-1.30 ; \\
p=0.418 \text { and mHealth } 0.98 \\
(0.62-1.57 ; p=0.945) \text { when } \\
\text { compared to control }\end{array}$ & $\begin{array}{l}\text { No statistical difference in } \\
\text { mean number of portions of } \\
\text { fruits and vegetables } \\
\text { consumed per day in both } \\
\text { PLA }(0.29(0.10-0.69 ; p= \\
0.143) \text { and } m \text { health }(-0.19 \\
(-0.53 \text { to } 0.15 ; p=0.27) \\
\text { groups compared to control }\end{array}$ & & & & $\begin{array}{l}\text { No significant difference } \\
\text { between the PLA and mHealth } \\
\text { group compared control }\end{array}$ & 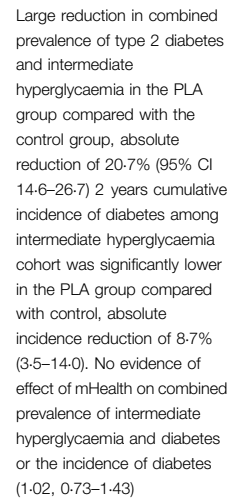 & \\
\hline
\end{tabular}


TABLE 2 | (Continued) Effect of community-based interventions on study outcomes, Community-based interventions for cardiovascular disease prevention in low- and middle-income countries: a systematic review,

\begin{tabular}{|c|c|c|c|c|c|c|c|c|c|}
\hline Author & Knowledge & $\begin{array}{l}\text { Physical } \\
\text { activity }\end{array}$ & Diet & Smoking & Alcohol & Body weight & Blood pressure & Blood glucose & Lipid profile \\
\hline $\begin{array}{l}\text { Nguyen, } \\
2012[41]\end{array}$ & & $\begin{array}{l}\text { In control group, physical } \\
\text { inactivity only significantly } \\
\text { changed in men and in } \\
\text { intervention group, significant } \\
\text { increase was in both men and } \\
\text { women. When both rroups } \\
\text { were compreared, significant } \\
\text { decrease was observed in } \\
\text { females }\end{array}$ & $\begin{array}{l}\text { Prevalence of salty diets } \\
\text { decreased significantly in } \\
\text { intervention group and was } \\
\text { unchanged in control group } \\
(p<0.001)\end{array}$ & $\begin{array}{l}\text { Prevalence of smoking did } \\
\text { not significantly change }\end{array}$ & $\begin{array}{l}\text { Reduction in alcohol } \\
\text { consumption in both } \\
\text { intervention and control } \\
\text { communities }(p<0.01)\end{array}$ & $\begin{array}{l}\text { Waist circumference and } \\
\text { waist hip ratio significantly } \\
\text { increased in both groups }(p< \\
0.01) \text {. Weight and BMI was } \\
\text { unchanged in intervention } \\
\text { group but significantly } \\
\text { reduced in control community } \\
\text { for BMl in both sexes }(p< \\
0.05 \text { ) and weight in women } \\
(p<0.01) \text {. Intervention group } \\
\text { registered significant increase } \\
\text { in weight, BMl and waist } \\
\text { circumference in women and } \\
\text { in men, BMl also increased }\end{array}$ & $\begin{array}{l}\text { SBP reduced in both groups } \\
\text { while DBP reduced only in the } \\
\text { intervention group }(p<0.01) \text {. } \\
\text { Comparing changes over time } \\
\text { between groups, SBP and DBP } \\
\text { significantly decreased in } \\
\text { intervention group. SBP vs DBP } \\
\text { ( } 3.3 \text { and } 4.7 \mathrm{mmHHg} \text { in women } \\
\text { vs. } 3.0 \text { and } 4.6 \mathrm{mmHHg} \text { in men, } \\
\text { respectively) }\end{array}$ & & \\
\hline $\begin{array}{l}\text { Chandraratne, } \\
2019 \text { [42] }\end{array}$ & & $\begin{array}{l}\text { No significant difference in } \\
\text { oddds of engaging in } \\
\text { recommended levels of leisure } \\
\text { time physical activity between } \\
\text { groups at at ollow-un }(p=0.15) \\
\text { and mean sedentary minutes } \\
(p=0.94)\end{array}$ & $\begin{array}{l}\text { Significantly higher odds of } \\
\text { consumingn one or more } \\
\text { serving/day of frutits (OR 1.71, } \\
1.11-2.65, p=0.02) \text { and } \\
\text { significantly lower odds of } \\
\text { consuming two or more } \\
\text { snacks per day (OR } 0.32, \\
0.21-0.48, p<0.001) \text { in } \\
\text { intervention than control } \\
\text { group. No significant } \\
\text { difference in the consumption } \\
\text { of vegetables or sugar } \\
\text { sweetened beverages } \\
\text { between groups }\end{array}$ & $\begin{array}{l}\text { No significant difference in } \\
\text { proportion of smokers in } \\
\text { both groups }\end{array}$ & $\begin{array}{l}\text { No significant difference in } \\
\text { proportion that consumed } \\
\text { more than two drinks per } \\
\text { day for men and one drink } \\
\text { for women }\end{array}$ & $\begin{array}{l}\text { Significantly lower mean body } \\
\text { weight }(61.8 \mathrm{~kg}[12.7) \text { ) and } \\
\text { BMI (24.4 } \mathrm{kg} / \mathrm{m} 2) \text { in } \\
\text { intervention than control } \\
\text { group (body weight } 64.0 \mathrm{~kg} \\
\text { [12.8], BMI } 25.5 \mathrm{~kg} / \mathrm{m} 2(4.7) \text {. } \\
\text { Mean difference: Body weight } \\
-2.83 \mathrm{~kg}(-3.31 \text { to }-2.35) \\
\text { and BMI (-1.12 kg/m2 }(-1.32 \\
\text { to - -0.94)). More pronounced } \\
\text { difference among overweight } \\
\text { persons (body weight } \\
-3.69 \mathrm{~kg} \text { and BMI }-1.50 \mathrm{~kg} / \\
\mathrm{m} 2, p<0.001)\end{array}$ & $\begin{array}{l}\text { No significant change in blood } \\
\text { pressure from baseline to } \\
\text { endline in both groups including } \\
\text { among hypertensives. Mean } \\
\text { difference in SBP }(-0.88) \text { and } \\
\text { DBP }(-0.94) \text { between } \\
\text { intervention and control groups } \\
\text { was also not significant }\end{array}$ & & \\
\hline Joshi, 2019 [43] & & & & $\begin{array}{l}\text { Proportion of individiduals who } \\
\text { use smokeless tobacco } \\
\text { significantly declined } \\
\text { between baseline and } \\
12 \text { months in both } \\
\text { intervention and control } \\
\text { groups and difference } \\
\text { between groups was } \\
\text { significant }(p=0.0001) \text {. } \\
\text { Proportion of individuals who } \\
\text { smoked decenined by } 4.1 \% \text { in } \\
\text { intervention arm and by } \\
2.6 \% \text { in control arm but the } \\
\text { difference between the two } \\
\text { groups was not statistically } \\
\text { signififiant }\end{array}$ & & & $\begin{array}{l}\text { Significant decline in SBP } \\
\text { (mmHg) from baseline in both } \\
\text { groups-controls } 130.3 \pm 21 \text { to } \\
128.3 \pm 15 \text {; intervention } 130.3 \pm \\
21 \text { to } 127.6 \pm 15(p<0.01 \text { for } \\
\text { before and after comparison) } \\
\text { but there was no difference } \\
\text { between the two groups at } \\
12 \text { months }(p=0.18)\end{array}$ & & \\
\hline $\begin{array}{l}\text { Gunawardena, } \\
2016[44]\end{array}$ & & 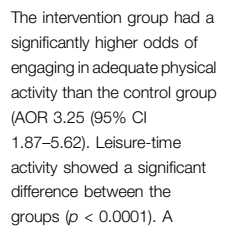 & $\begin{array}{l}\text { No significant difiference in } \\
\text { individual-level food } \\
\text { consumption between the } \\
\text { two groups including fruits } \\
\text { and vegetables consumption } \\
\text { after the intervention, but the } \\
\text { intervention group showed a } \\
\text { significant decrease in } \\
\text { household-level purchase of }\end{array}$ & & & $\begin{array}{l}\text { Intervention group had a } \\
\text { significantly lower mean of } \\
\text { weight and BMl than did } \\
\text { control group }(p<0.0001) \text {; } \\
\text { mean }(95 \% \mathrm{Cl} \text { ) effect between } \\
\text { groups was }-2.49(-3.38 \text { to } \\
-1.60) \mathrm{kg} \text { and }-0.99(-1.40 \\
\text { to }-0.58) \mathrm{kg} / \mathrm{m} 2\end{array}$ & & & \\
\hline
\end{tabular}


TABLE 2 | (Continued) Effect of community-based interventions on study outcomes, Community-based interventions for cardiovascular disease prevention in low- and middle-income countries: a systematic review,

\begin{tabular}{|c|c|c|c|c|c|c|c|c|c|}
\hline Author & Knowledge & $\begin{array}{l}\text { Physical } \\
\text { activity }\end{array}$ & Diet & Smoking & Alcohol & Body weight & Blood pressure & Blood glucose & Lipid profile \\
\hline Joshi, 2012 [45] & $\begin{array}{l}\text { No statistically significant } \\
\text { effect on knowledge about } 6 \\
\text { lifistly factors affecting CVD } \\
\text { risk }(p=0.15)\end{array}$ & $\begin{array}{l}\text { No significant difference in } \\
\text { mean number of days of light } \\
\text { and medium/heavy physical } \\
\text { activity }(p=0.78)\end{array}$ & $\begin{array}{l}\text { Individuals in the villages who } \\
\text { received a health promotion } \\
\text { intervention were significantly } \\
\text { more likely to avoid } \\
\text { consumption of oily foods } \\
(p=0.01) \text {. No change in } \\
\text { mean no. of days eat fruit, } \\
\text { green leafy vegetables and } \\
\text { salt consumption }\end{array}$ & $\begin{array}{l}\text { No significant change in } \\
\text { proportion not currently } \\
\text { using tobacco }(p=0.74)\end{array}$ & & $\begin{array}{l}\text { No change in mean body } \\
\text { mass index, mean waist } \\
\text { circumference }\end{array}$ & $\begin{array}{l}\text { No significant change in mean } \\
\text { systolic, diastolic between } \\
\text { intervention and control groups } \\
\text { following intervention }\end{array}$ & & \\
\hline Lu, $2015[46]$ & $\begin{array}{l}\text { Improvements in } \\
\text { hypertension-related } \\
\text { knowledge score registered in } \\
\text { all groups but greatest in } \\
\text { group } 3 \text {, then } 2 \text { and } 1\end{array}$ & $\begin{array}{l}\text { Regular physical activity } \\
\text { increased in all groups } \\
\text { progressively from group } 1 \\
\text { (seff-learning reading) to } 2 \\
\text { (regular ididactic elcture) and } \\
\text { highest in } 3 \text { (interactive } \\
\text { education workshops) }\end{array}$ & $\begin{array}{l}\text { Adherence to appropriate salt } \\
\text { intake was progressively } \\
\text { greater from group } 1 \text { to } 3\end{array}$ & $\begin{array}{l}\text { No significant difference in } \\
\text { proportion of current } \\
\text { smokers in all groups }\end{array}$ & $\begin{array}{l}\text { No significant difference in } \\
\text { proportion of alcohol } \\
\text { drinkers in all groups }\end{array}$ & $\begin{array}{l}\text { BMI decreased significantly in } \\
\text { group } 1 \text { (self-reading) and } 3 \\
\text { (interactive education } \\
\text { workshop) - largest reduction } \\
\text { and waist and hip ratio } \\
\text { decreased significantly only in } \\
\text { group 3. The difference } \\
\text { across groups was } \\
\text { statistically significant }\end{array}$ & $\begin{array}{l}\text { Proportion of subjects with } \\
\text { normalized BP increased in } \\
\text { group } 2(41.2 \%-63.2 \%, p< \\
0.001) \text { and more substantially in } \\
\text { group } 3(40.2 \%-86.3 \%, p< \\
\text { 0.001). No significant change in } \\
\text { group } 1\end{array}$ & & 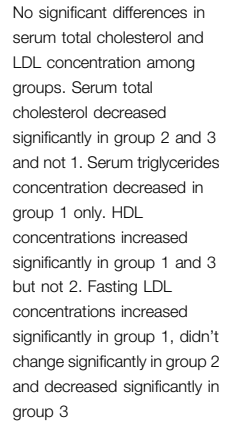 \\
\hline Lv, $2014[47]$ & $\begin{array}{l}\text { Tobacco related knowledge } \\
\text { significantly improved in } \\
\text { intervention compared to } \\
\text { control. Diet and physical } \\
\text { activity knowledge and beliefs } \\
\text { decreased in intervention } \\
\text { compared to control }\end{array}$ & $\begin{array}{l}\text { The metabolic equivalent of } \\
\text { physical activity significantly } \\
\text { increased in intevention }(p= \\
0.023 \text { compared to control } \\
(p=0.201)\end{array}$ & $\begin{array}{l}\text { The fruit and vegetable } \\
\text { consumption score } \\
\text { significantly increased in both } \\
\text { the intervention (24.84 vs } \\
25.97,, 0=0.036 \text { a and } \\
\text { comparison (24.25 vs } 26.67 \text {, } \\
p<0.001) \text { areas but } \\
\text { differencer between the two } \\
\text { not significant }\end{array}$ & $\begin{array}{l}\text { A statisticically significant } \\
\text { decline in the prevalence of } \\
\text { smoking observed in } \\
\text { intervention }(25.2 \% \text { vs } \\
18.7 \%, p<0.001) \text { compared } \\
\text { with the comparison area } \\
(18.0 \% \text { vs } 16.4 \%, p=0.343) \text {. } \\
\text { Statistically significant } \\
\text { difference in prevalence also } \\
\text { observed among men }\end{array}$ & & & & & \\
\hline Chao, 2012 [48] & & $\begin{array}{l}\text { Intervention group } \\
\text { demonstrated significant } \\
\text { improvement in physical } \\
\text { activity duration per week }\end{array}$ & $\begin{array}{l}\text { Intervention group } \\
\text { demonstrated significaant } \\
\text { improvement in diet score } \\
\text { compared to control group }\end{array}$ & & & $\begin{array}{l}\text { Waist to hip ratio significantly } \\
\text { reduced in the intervention } \\
\text { group compared to controls }\end{array}$ & $\begin{array}{l}\text { Systollic blood pressure } \\
\text { significantly reduced in the } \\
\text { intervention compared to } \\
\text { controls. No difference } \\
\text { observed in diastollic blood } \\
\text { pressure between groups }\end{array}$ & $\begin{array}{l}\text { Fasting blood sugar } \\
\text { significantly reduced in the } \\
\text { intervention group compared } \\
\text { to controls }\end{array}$ & $\begin{array}{l}\text { No difference observed in } \\
\text { lipidemia patients triglyceride } \\
\text { between groups }\end{array}$ \\
\hline Huang, 2011 [49] & $\begin{array}{l}\text { Statistically significant } \\
\text { changes in hypertension } \\
\text { knowledge }(p<0.05)\end{array}$ & $\begin{array}{l}\text { The percentage of } \\
\text { particicants physically active } \\
\text { increased from } 43.3 \text { to } 59.7 \% \\
\text { in the intervention group and } \\
43.7-70.2 \% \text { in the control } \\
\text { group, both statistically } \\
\text { significant }\end{array}$ & $\begin{array}{l}\text { Participants in intervention } \\
\text { group exhibited a signifificantly } \\
\text { greater improvement in } \\
\text { dietary habits including } \\
\text { reducing salty food intake } \\
(13.6 \% \text { vs. } 21.7 \%) \text {, fat intake } \\
(22.9 \% \text { vs. } 31.9 \%) \\
\text { comparison with those in the } \\
\text { control group. Consumption } \\
\text { of pickled food reduced in } \\
\text { intervention and increased in } \\
\text { controlo group though not } \\
\text { statistically significant. } \\
\text { Reduction in consumption of } \\
\text { pickled food registered in } \\
\text { both groups but difference } \\
\text { not significant }(p=0.641)\end{array}$ & $\begin{array}{l}\text { Smoking reduced in the } \\
\text { intervention group } \\
(29.5-26.0 \%) \text { and increased } \\
\text { in the control group } \\
\text { (23.9-2.7.0\%) and the } \\
\text { difference was not } \\
\text { statistically significant }\end{array}$ & $\begin{array}{l}\text { Intervention group } \\
\text { participants exhibited a } \\
\text { significantly greater } \\
\text { improvement in alcohol } \\
\text { consumption compared } \\
\text { with control group }(9.6 \%) \text { vs. } \\
18.0 \%, p<0.05)\end{array}$ & & $\begin{array}{l}\text { A significant reduction in the } \\
\text { prevalence rate of hypertension } \\
\text { in only the intervention group, } \\
\text { which was from } 35.4 \text { to } 22.5 \% \text {. } \\
\text { No change in the prevalence of } \\
\text { hypertension in the control } \\
\text { group. Significant increase in } \\
\text { treatment }(p<0<05 \text { and control } \\
\text { rates of } h y p \text { rertensive patients in } \\
\text { both groups }(p<0.05 \text { and } \\
\text { between group in favor of the } \\
\text { control group }(p<0.05)\end{array}$ & & \\
\hline
\end{tabular}


TABLE 2 | (Continued) Effect of community-based interventions on study outcomes, Community-based interventions for cardiovascular disease prevention in low- and middle-income countries: a systematic review, 2000-2019.

\begin{tabular}{|c|c|c|c|c|c|c|c|c|c|}
\hline Author & Knowledge & $\begin{array}{l}\text { Physical } \\
\text { activity }\end{array}$ & Diet & Smoking & Alcohol & Body weight & Blood pressure & Blood glucose & Lipid profile \\
\hline Zhang, 2018 [50] & & $\begin{array}{l}\text { Comparing intervention and } \\
\text { control groups, adherence to } \\
\text { physical activity was } \\
\text { significant at both } 12 \text { and } \\
24 \text { months }\end{array}$ & $\begin{array}{l}\text { Comparing intervention and } \\
\text { control groups, adherence to } \\
\text { high diet score was } \\
\text { significant at both } 12 \text { and } \\
24 \text { months }\end{array}$ & $\begin{array}{l}\text { Adherence to non-smoking } \\
\text { among intervention } \\
\text { compared to control was not } \\
\text { significant at both } 12 \text { and } \\
24 \text { months }\end{array}$ & $\begin{array}{l}\text { Adherence to moderate } \\
\text { alcohol use among } \\
\text { intervention compared to } \\
\text { control was not significant at } \\
\text { both } 12 \text { and } 24 \text { months }\end{array}$ & $\begin{array}{l}\text { Average waist circumference } \\
\text { lower in intervention group but } \\
\text { not statistically significant both } \\
\text { at } 12 \text { and } 24 \text { months }\end{array}$ & $\begin{array}{l}\text { Average systollic and diastollic } \\
\text { BP significantly lower in } \\
\text { intervention group compared to } \\
\text { control both at } 12 \text { and } \\
24 \text { months }\end{array}$ & $\begin{array}{l}\text { Fasting plasma glucose } \\
\text { significantly lower in } \\
\text { intervention group compared } \\
\text { to control both at } 12 \text { and } \\
24 \text { months }\end{array}$ & $\begin{array}{l}\text { Total cholesterol significantly } \\
\text { lower in intervention group } \\
\text { than control both at } 12 \text { and } \\
24 \text { months }\end{array}$ \\
\hline $\begin{array}{l}\text { Ibrahim, } \\
2016[51]\end{array}$ & & $\begin{array}{l}\text { Greater proportion of } \\
\text { participants from the Co- } \\
\text { HELP group met the clinical } \\
\text { recommended target physical } \\
\text { activity of }>600 \mathrm{METS} / \mathrm{min} / \mathrm{wk} \\
(60.7 \% \text { vs } 32.2 \%, p<0.001) \\
\text { compared to the usual care } \\
\text { group }\end{array}$ & $\begin{array}{l}\text { Intervention group showed a } \\
\text { greater percentage of } \\
\text { participants (13.9\%) who met } \\
\text { the dietary aims (to reduce } \\
20 \pm 25 \mathrm{kccal} / \mathrm{kg} \text { energy } \\
\text { intiake) as compared to usual } \\
\text { care group }(9.6 \% \text { ) but not } \\
\text { statistically significant }\end{array}$ & & & $\begin{array}{l}\text { Greater proportion of } \\
\text { intervention participants met } \\
\text { the clinical recommended } \\
\text { target of } 5 \% \text { or more weight } \\
\text { loss from the initial weight } \\
(24.6 \% \text { vs. } 3.4 \%, p<0.001) \text {. } \\
\text { Change in waist } \\
\text { circumference was }-2.44 \mathrm{~cm} \\
(-4.75 \text { to }-0.12, p<0.05)\end{array}$ & $\begin{array}{l}\text { At } 12 \text { months, SBP reduced in } \\
\text { intervention compared to } \\
\text { control group but not significant } \\
(-1.71(-3.97 \text { to } 0.56)) \text {. DBP } \\
\text { changed by }-2.63 \mathrm{mmHg} \\
(-3.79 \text { to }-1.48, p<0.01) \\
\text { compared to the control group }\end{array}$ & $\begin{array}{l}\text { Analysis of between-groups at } \\
12 \text { months (mean difference, } \\
95 \% \mathrm{CI} \text { ) reveaeled that the Co- } \\
\text { HELP participants' mean } \\
\text { fasting plasma glucose } \\
\text { reduced by }-0.40 \mathrm{mmol} / \mathrm{L} \\
(-0.51 \text { to }-0.28, p<0.001) \text {, } \\
2 \mathrm{~h} \text { post glucose by } \\
-0.58 \mathrm{mmol} / \mathrm{L}(-0.91 \text { to } \\
-0.24, p<0.001), \mathrm{HbAAC} \text { by } \\
-0.24 \%(-0.34 \text { to }-0.15, p< \\
0.001)\end{array}$ & $\begin{array}{l}\text { HDL cholesterol increased by } \\
0.12 \text { mmoll }(0.05-0.19, p< \\
0.01) \text {, compared to the usual } \\
\text { care group }\end{array}$ \\
\hline $\begin{array}{l}\text { Mcalister, } \\
2000[52]\end{array}$ & & & & $\begin{array}{l}\text { Cessation rates were } 7-26 \% \\
\text { in pitkäranta and } 1-2 \% \text { in the } \\
\text { comparison area }(p<0.05)\end{array}$ & & & & & \\
\hline Aung, 2019 [53] & & & & $\begin{array}{l}\text { Intervention participants } \\
(25.62 \%) \text { achieved a } \\
\text { significantly higher smoking } \\
\text { cessation rate than the } \\
\text { control participants } \\
\left(\begin{array}{l}11.32 \%), \text { adjusted analysis } \\
\text { (AOR } 2.95, p<0.001)\end{array}\right.\end{array}$ & & & & & \\
\hline Latina, 2020 [54] & & $\begin{array}{l}\text { No significant difference } \\
\text { between the two groups in } \\
\text { exercise }\end{array}$ & & $\begin{array}{l}\text { No significant change in } \\
\text { tobacco use between } \\
\text { groups }\end{array}$ & & & & $\begin{array}{l}\text { At one-year of follow-up, the } \\
\text { overall FBS was significantly } \\
\text { different between the peer } \\
\text { group intervention and control } \\
\text { groups }[9.1 \text { (SD 2.7) vs. } 8.5 \\
\text { (SD 2.6), } p=0.028\end{array}$ & \\
\hline $\begin{array}{l}\text { Anthony, } \\
2015 \text { [55] }\end{array}$ & & $\begin{array}{l}\text { No significant difference } \\
\text { between intervention and } \\
\text { control in change in physical } \\
\text { activity }\end{array}$ & $\begin{array}{l}\text { The proportion eating five } \\
\text { portions of frut and } \\
\text { vegetalabs increased in } \\
\text { intervention compared to } \\
\text { control group (6.9\% vs. } \\
1.5 \%, p<0.001) \text {. Salt intake } \\
\text { increased in both groups } \\
\text { more in the control compared } \\
\text { to the intervention group }(p= \\
0.014)\end{array}$ & $\begin{array}{l}\text { Prevalence of tobacco use } \\
\text { significantly reduced in men } \\
(6.0 \% \text { vs. } 2.6 \%, p<0.001) \text { in } \\
\text { intervention compared with } \\
\text { control. In women, tobacco } \\
\text { use slightly increased in both } \\
\text { groups with no difference }\end{array}$ & & $\begin{array}{l}\text { No significant difference } \\
\text { between intervention and } \\
\text { control in change in } \\
\text { overweight }\end{array}$ & & & \\
\hline $\begin{array}{l}\text { Neupane, } \\
2018[56]\end{array}$ & & $\begin{array}{l}\text { No significant differences } \\
\text { between intervention and } \\
\text { control groups at follow-up in } \\
\text { proportions of people who } \\
\text { had low physical activity (OR = } \\
0.77,95 \% \text { Cl } 0 \text { 24-2.45) }\end{array}$ & 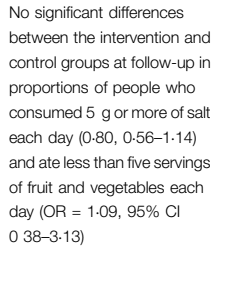 & $\begin{array}{l}\text { No significant differences } \\
\text { between the intervention and } \\
\text { control groups at follow-up in } \\
\text { proportions of people who } \\
\text { smoked daily (RR 0.79, 95\% } \\
\text { CI 0 06-1.37) }\end{array}$ & $\begin{array}{l}\text { No significant differences } \\
\text { between groups at follow-up } \\
\text { in proportions of people who } \\
\text { consumed harmful amounts } \\
\text { of alcohol (OR 1.07, } 95 \% \mathrm{Cl} \\
061-1.90)\end{array}$ & & $\begin{array}{l}\text { Mean } \mathrm{SBP} \text { at } 1 \text { year was } \\
\text { significantly lower in the } \\
\text { intervention group than in the } \\
\text { control group for all cohorts: } \\
\text { (Difference }-2.28 \mathrm{~mm} \mathrm{Hg}(95 \% \\
\mathrm{Cl}-3.77 \text { to }-0.79, p=0.003) \text { for } \\
\text { normotensive participants, } \\
-3.08 \mathrm{~mm} \mathrm{Hg}(-5.58 \text { to }-0.59 \text {, } \\
p=0.015) \text { for prehypertensive, } \\
\text { and }-4.90 \mathrm{~mm} \mathrm{Hg}(-7.78 \text { to } \\
-2.00, p=0.001) \text { for } \\
\text { hypertensive participants }\end{array}$ & & \\
\hline
\end{tabular}

No significant difference

between the two groups

No significant difference between intervention and
control in change in phys control
No significant dififerences control groups at follow-L 77, 95\% Cl 0 24-2.45)
cant differences ween the intervention and tervention participants sgnificantly higher smokning control participants intervention compared to increased in both groups to the intervention group $(0=$ 
could have provided recent studies. However, such abstracts sometimes do not contain adequate information and their results may be inconclusive. The heterogeneity of the outcome measures also meant that a meta-analysis was not possible. Nevertheless, this systematic review provides evidence on the effectiveness of community-based interventions for CVD prevention in LMICs and their effectiveness in improving knowledge and uptake of healthy lifestyles in addition to changing metabolic risk factors essential for CVD prevention.

\section{Conclusion}

This review found several community-based interventions implemented for CVD prevention in LMICs which significantly influenced knowledge about CVD and risk factors, and changes in physical activity and dietary behaviors for CVD prevention. However, evidence on reducing smoking and alcohol consumption were inconsistent necessitating further research. Regarding the CVD metabolic risk factors, communitybased interventions significantly impacted blood pressure and blood sugar measurements. The most effective interventions utilized community mobilization, health education and information sharing, individual or group counseling, and trainings of providers. Evidence from this review can inform policy makers in decision-making and prioritizing evidencebased interventions for CVD prevention in LMICs.

\section{AUTHOR CONTRIBUTIONS}

RN, RW, HB and GM conceptualized this review. RN, HH, RW, $\mathrm{DM}, \mathrm{FN}, \mathrm{SA}, \mathrm{HB}, \mathrm{GM}$ contributed to the review protocol and were

\section{REFERENCES}

1. World Health Organization. Cardiovascular Diseases (CVDs). (2017). Available from: http://www.who.int/mediacentre/factsheets/fs317/en/

2. Gaziano, TA, Bitton, A, Anand, S, Abrahams-Gessel, S, and Murphy, A. Growing Epidemic of Coronary Heart Disease in Low- and Middle-Income Countries. Curr Probl Cardiol (2010). 35(2):72-115. doi:10.1016/j.cpcardiol.2009.10.002

3. Ng, M, Fleming, T, Robinson, M, Thomson, B, Graetz, N, and Margono, C. Global, Regional, and National Prevalence of Overweight and Obesity in Children and Adults during 1980-2013: a Systematic Analysis for the Global Burden of Disease Study 2013. Lancet (2014). 384(9945):766-81. doi:10.1016/ S0140-6736(14)60460-8

4. Aboderin, I, Kalache, A, Ben Shlomo, Y, Lynch, J, Yajnik, C, and Kuh, D. Life Course Perspectives on Coronary Heart Disease, Stroke and Diabetes: Key Issues and Implications for Policy and Research. Geneva, Switzerland: World Health Organisation (2002).

5. Sarki, AM, Nduka, CU, Stranges, S, Kandala, N-B, and Uthman, OA. Prevalence of Hypertension in Low-And Middle-Income Countries: a Systematic Review and Meta-Analysis. Medicine (2015). 94(50). doi:10. 1097/md.0000000000001959

6. Bosu, WK, Reilly, ST, Aheto, JMK, and Zucchelli, E. Hypertension in Older Adults in Africa: A Systematic Review and Meta-Analysis. PloS one (2019). 14(4):e0214934. doi:10.1371/journal.pone.0214934

7. World Health Organization. Prevention of Cardiovascular Disease. Geneva, Switzerland: World Health Organization (2007).

8. Merzel, C, and D'Afflitti, J. Reconsidering Community-Based Health Promotion: Promise, Performance, and Potential. Am J Public Health (2003). 93(4):557-74. doi:10.2105/ajph.93.4.557 involved in conducting the review and supervising the process. RN prepared the original draft of the manuscript. HH, RW, DM, FN, SA, HB, GM thoroughly reviewed and edited the manuscript. All authors read and approved the final version of the manuscript.

\section{FUNDING}

This work was supported by the SPICES project in Uganda which received funding from the European Commission through the Horizon 2020 research and innovation action grant agreement No 733356 to implement and evaluate a comprehensive CVD prevention program in five settings: a rural and semi-urban community in a low-income country (Uganda), middle income (South Africa) and vulnerable groups in three highincome countries (Belgium, France and United Kingdom). The funder had no role in the design, decision to publish, or preparation of the manuscript.

\section{CONFLICT OF INTEREST}

The authors declare that the research was conducted in the absence of any commercial or financial relationships that could be construed as a potential conflict of interest.

\section{ACKNOWLEDGMENTS}

The authors wish to thank Isaac Ddumba for his contribution to the review protocol.

9. Maya-Jariego, I, and Holgado, D. Community Interventions. Introduction to Community Psychology, Montreal, Canada: Pressbooks (2019).

10. Parker, DR, and Assaf, AR. Community Interventions for Cardiovascular Disease. Prim Care Clin Off Pract (2005). 32(4):865-81. doi:10.1016/j.pop. 2005.09.012

11. Elder, JP, Schmid, TL, Dower, P, and Hedlund, S. Community Heart Health Programs: Components, Rationale, and Strategies for Effective Interventions. J Public Health Pol (1993). 14(4):463-79. doi:10.2307/3342878

12. Cecchini, M, Sassi, F, Lauer, JA, Lee, YY, Guajardo-Barron, V, and Chisholm, D. Tackling of Unhealthy Diets, Physical Inactivity, and Obesity: Health Effects and Cost-Effectiveness. The Lancet. (2010). 376(9754):1775-84. doi:10.1016/ S0140-6736(10)61514-0

13. Checkley, W, Ghannem, H, Irazola, V, Kimaiyo, S, Levitt, NS, and Miranda, JJ. Management of NCD in Low- and Middle-Income Countries. Glob Heart. (2014). 9(4):431-43. doi:10.1016/j.gheart.2014.11.003

14. Rodrigues, AL, Ball, J, Ski, C, Stewart, S, and Carrington, MJ. A Systematic Review and Meta-Analysis of Primary Prevention Programmes to Improve Cardio-Metabolic Risk in Non-urban Communities. Prev Med (2016). 87: 22-34. doi:10.1016/j.ypmed.2016.02.011

15. Lawlor, ER, Bradley, DT, Cupples, ME, and Tully, MA. The Effect of Community-Based Interventions for Cardiovascular Disease Secondary Prevention on Behavioural Risk Factors. Prev Med (2018). 114:24-38. doi:10.1016/j.ypmed.2018.05.019

16. Brown, T, Smith, S, Bhopal, R, Kasim, A, and Summerbell, C. Diet and Physical Activity Interventions to Prevent or Treat Obesity in South Asian Children and Adults: a Systematic Review and Meta-Analysis. Ijerph (2015). 12(1):566-94. doi:10.3390/ijerph120100566

17. Beauchamp, A, Backholer, K, Magliano, D, and Peeters, A. The Effect of Obesity Prevention Interventions According to Socioeconomic Position: 
a Systematic Review. Obes Rev (2014). 15(7):541-54. doi:10.1111/obr. 12161

18. Khetan, AK, Purushothaman, R, Chami, T, Hejjaji, V, Madan Mohan, SK, and Josephson, RA. The Effectiveness of Community Health Workers for CVD Prevention in LMIC. Glob Heart. (2017). 12(3):233-43.e6. doi:10.1016/j. gheart.2016.07.001

19. van de Vijver, S., Oti, S., Addo, J., de Graft-Aikins, A., and Agyemang, C. Review of community-based interventions for prevention of cardiovascular diseases in low- and middle-income countries. Ethnicity and Health. (2012). 17(6):651-676. doi:10.1080/13557858.2012.754409

20. Moher, D, Shamseer, L, Clarke, M, Ghersi, D, Liberati, A, and Petticrew, M. Preferred Reporting Items for Systematic Review and Meta-Analysis Protocols (PRISMA-P) 2015 Statement. Syst Rev (2015). 4(1):1. doi:10.1186/2046-40534-1

21. Ouzzani, M, Hammady, H, Fedorowicz, Z, and Elmagarmid, A. Rayyan-a Web and mobile App for Systematic Reviews. Syst Rev (2016). 5(1):210. doi:10. 1186/s13643-016-0384-4

22. Moher, D., Liberati, A., Tetzlaff, J., and Altman, D. G. Preferred reporting items for systematic reviews and meta-analyses: the PRISMA statement. Bmj. (2009). 339:b2535. doi:10.1136/bmj.b2535

23. Sterne, JAC, Savović, J, Page, MJ, Elbers, RG, Blencowe, NS, and Boutron, I. RoB 2: a Revised Tool for Assessing Risk of Bias in Randomised Trials. BMJ (2019). 366:14898. doi:10.1136/bmj.14898

24. Sterne, JA, Hernán, MA, Reeves, BC, Savović, J, Berkman, ND, and Viswanathan, M. ROBINS-I: a Tool for Assessing Risk of Bias in Nonrandomised Studies of Interventions. Bmj (2016). 355:i4919. doi:10.1136/ bmj.i4919

25. Baghaei, A, Sarrafzadegan, N, Rabiei, K, Gharipour, M, Tavasoli, AA, and Shirani, S. Research paperHow Effective Are Strategies for Non-communicable Disease Prevention and Control in a High Risk Population in a Developing Country? Isfahan Healthy Heart Programme. aoms (2010). 1(1):24-31. doi:10. 5114/aoms.2010.13503

26. Kelishadi, R, Sarrafzadegan, N, Sadri, GH, Pashmi, R, Mohammadifard, N, and Tavasoli, AA. Short-Term Results of a Community-Based Program on Promoting Healthy Lifestyle for Prevention and Control of Chronic Diseases in a Developing Country Setting. Asia Pac J Public Health (2011). 23(4):518-33. doi:10.1177/1010539509348241

27. Rabiei, K, Kelishadi, R, Sarrafzadegan, N, Sadri, G, and Amani, A. Short-term Results of Community-Based Interventions for Improving Physical Activity: Isfahan Healthy Heart Programme. aoms (2010). 1(1):32-9. doi:10.5114/aoms. 2010.13504

28. Sarrafzadegan, N, Kelishadi, R, Esmaillzadeh, A, Mohammadifard, N, Rabiei, $\mathrm{K}$, and Roohafza, H. Do lifestyle Interventions Work in Developing Countries? Findings from the Isfahan Healthy Heart Program in the Islamic Republic of Iran. Bull World Health Org (2009). 87:39-50. doi:10.2471/blt.07.049841

29. Sarrafzadegan, N, Sadeghi, M, Tavassoli, A, Mohseni, M, Alikhasi, H, and Rabiei, K. Sex Differences in the Impact of a Community-Based Program for Non-communicable Disease Prevention: The Isfahan Healthy Heart Program (IHHP). J Public Health (2009). 17(4):257-63. doi:10.1007/s10389-008-0240-7

30. Azizi, F, Mirmiran, P, Momenan, AA, Hadaegh, F, Moeini, AH, and Hosseini, F. The Effect of Community-Based Education for Lifestyle Intervention on the Prevalence of Metabolic Syndrome and its Components: Tehran Lipid and Glucose Study. Int J Endocrinol Metab (2013). 11(3):145. doi:10.5812/ijem. 5443

31. Mirmiran, P, Ramezankhani, A, Hekmatdoost, A, and Azizi, F. Effect of Nutrition Intervention on Non-communicable Disease Risk Factors Among Tehranian Adults: Tehran Lipid and Glucose Study. Ann Nutr Metab (2008). 52(2):91-5. doi:10.1159/000121364

32. van de Vijver, S, Oti, SO, Gomez, GB, Agyemang, C, Egondi, T, and van Charante, EM. Impact Evaluation of a Community-Based Intervention for Prevention of Cardiovascular Diseases in the Slums of Nairobi: the SCALE-UP Study. Glob Health Action (2016). 9(1):30922. doi:10.3402/gha.v9.30922

33. Daivadanam, M, Wahlström, R, Ravindran, TS, Sarma, PS, Sivasankaran, S, and Thankappan, K. Changing Household Dietary Behaviours through Community-Based Networks: A Pragmatic Cluster Randomized Controlled Trial in Rural Kerala, India. PloS one (2018). 13(8):e0201877. doi:10.1371/ journal.pone.0201877
34. Thankappan, KR, Sathish, T, Tapp, RJ, Shaw, JE, Lotfaliany, M, and Wolfe, R. A Peer-Support Lifestyle Intervention for Preventing Type 2 Diabetes in India: A Cluster-Randomized Controlled Trial of the Kerala Diabetes Prevention Program. PLoS Med (2018). 15(6):e1002575. doi:10.1371/journal.pmed.1002575

35. Jayakrishnan, R, Uutela, A, Mathew, A, Auvinen, A, Mathew, PS, and Sebastian, P. Smoking Cessation Intervention in Rural kerala, India: Findings of a Randomised Controlled Trial. Asian Pac J Cancer Prev (2013). 14(11):6797-802. doi:10.7314/apjcp.2013.14.11.6797

36. Ramachandran, A, Snehalatha, C, Mary, S, Mukesh, B, Bhaskar, AD, and Vijay, V. The Indian Diabetes Prevention Programme Shows that Lifestyle Modification and Metformin Prevent Type 2 Diabetes in Asian Indian Subjects with Impaired Glucose Tolerance (IDPP-1). Diabetologia (2006). 49(2):289-97. doi:10.1007/s00125-005-0097-z

37. Ramachandran, A, Snehalatha, C, Ram, J, Selvam, S, Simon, M, and Nanditha, A. Effectiveness of mobile Phone Messaging in Prevention of Type 2 Diabetes by Lifestyle Modification in Men in India: a Prospective, Parallel-Group, Randomised Controlled Trial. Lancet Diabetes Endocrinol (2013). 1(3): 191-8. doi:10.1016/s2213-8587(13)70067-6

38. Nishtar, S, Badar, A, Kamal, MU, lqbal, A, Bajwa, R, and Shah, T. The Heartfile Lodhran CVD Prevention Project- End of Project Evaluation. Promot Edu (2007). 14(1):17-27. doi:10.1177/175797590701400103

39. Jafar, TH, Hatcher, J, Poulter, N, Islam, M, Hashmi, S, and Qadri, Z. Community-Based Interventions to Promote Blood Pressure Control in a Developing Country. Ann Intern Med (2009). 151(9):593-601. doi:10.7326/ 0003-4819-151-9-200911030-00004

40. Fottrell, E, Ahmed, N, Morrison, J, Kuddus, A, Shaha, SK, and King, C. Community Groups or mobile Phone Messaging to Prevent and Control Type 2 Diabetes and Intermediate Hyperglycaemia in Bangladesh (DMagic): a Cluster-Randomised Controlled Trial. Lancet Diabetes Endocrinol (2019). 7(3):200-12. doi:10.1016/s2213-8587(19)30001-4

41. Nguyen, QN, Pham, ST, Nguyen, VL, Weinehall, L, Wall, S, and Bonita, R. Effectiveness of Community-Based Comprehensive Healthy Lifestyle Promotion on Cardiovascular Disease Risk Factors in a Rural Vietnamese Population: a Quasi-Experimental Study. BMC Cardiovasc Disord (2012). 12(1):56. doi:10.1186/1471-2261-12-56

42. Chandraratne, N, Yamaguchi, M, Indrawansa, S, Gunawardena, N, Kuwahara, $\mathrm{K}$, and Islam, Z. The Effect of Youths as Change Agents on Cardiovascular Disease Risk Factors Among Adult Neighbours: a Cluster Randomised Controlled Trial in Sri Lanka. BMC public health (2019). 19(1):893. doi:10. 1186/s12889-019-7142-1

43. Joshi, R, Agrawal, T, Fathima, F, Usha, T, Thomas, T, and Misquith, D. Cardiovascular Risk Factor Reduction by Community Health Workers in Rural India: A Cluster Randomized Trial. Am Heart J (2019). 216:9-19. doi:10. 1016/j.ahj.2019.06.007

44. Gunawardena, N, Kurotani, K, Indrawansa, S, Nonaka, D, Mizoue, T, and Samarasinghe, D. School-based Intervention to Enable School Children to Act as Change Agents on Weight, Physical Activity and Diet of Their Mothers: a Cluster Randomized Controlled Trial. Int J Behav Nutr Phys Activity (2016). 13(1):45. doi:10.1186/s12966-016-0369-7

45. Joshi, R., Chow, C. K., Raju, P. K., Raju, K. R., Gottumukkala, A. K., Reddy, K. S., MacMahon, S., Heritier, S., Li, Q., Dandona, R., and Neal, B. The Rural Andhra Pradesh Cardiovascular Prevention Study (RAPCAPS). Journal of the American College of Cardiology. (2012). 59(13):1,188-1,196. doi:10.1016/j.jacc. 2011.10.901

46. Lu, C-H, Tang, S-T, Lei, Y-X, Zhang, M-Q, Lin, W-Q, and Ding, S-H. Community-based Interventions in Hypertensive Patients: a Comparison of Three Health Education Strategies. BMC public health (2015). 15(1):33. doi:10. 1186/s12889-015-1401-6

47. Lv, J, Liu, Q-M, Ren, Y-J, He, P-P, Wang, S-F, and Gao, F. A CommunityBased Multilevel Intervention for Smoking, Physical Activity and Diet: ShortTerm Findings from the Community Interventions for Health Programme in Hangzhou, China. J Epidemiol Community Health (2014). 68(4):333-9. doi:10. 1136/jech-2013-203356

48. Chao, J, Wang, Y, Xu, H, Yu, Q, Jiang, L, and Tian, L. The Effect of Community-Based Health Management on the Health of the Elderly: a Randomized Controlled Trial from China. BMC Health Serv Res (2012). 12(1):449. doi:10.1186/1472-6963-12-449 
49. Huang, S, Hu, X, Chen, H, Xie, D, Gan, X, and Wu, Y. The Positive Effect of an Intervention Program on the Hypertension Knowledge and Lifestyles of Rural Residents over the Age of 35 Years in an Area of China. Hypertens Res (2011). 34(4):503-8. doi:10.1038/hr.2010.265

50. Zhang, M, Chao, J, Li, D, Gu, J, Chen, W, and Xu, H. The Effect of OlderPerson Centered and Integrated Health Management Model on Multiple Lifestyle Behaviors: A Randomized Controlled Trial from China. Arch Gerontol Geriatr (2018). 79:45-51. doi:10.1016/j.archger.2018.07.012

51. Ibrahim, N, Ming Moy, F, Awalludin, IAN, Mohd Ali, Z, and Ismail, IS. Effects of a Community-Based Healthy Lifestyle Intervention Program (Co-HELP) Among Adults with Prediabetes in a Developing Country: a QuasiExperimental Study. PloS one (2016). 11(12):e0167123. doi:10.1371/journal. pone. 0167123

52. McAlister, AL, Gumina, T, Urjanheimo, E-L, Laatikainen, T, Uhanov, M, and Oganov, R. Promoting Smoking Cessation in Russian Karelia: a 1-year Community-Based Program with Quasi-Experimental Evaluation. Health Promot Int (2000). 15(2):109-12. doi:10.1093/heapro/15.2.109

53. Aung, MN, Yuasa, M, Moolphate, S, Lorga, T, Yokokawa, H, and Fukuda, H. Effectiveness of a New Multi-Component Smoking Cessation Service Package for Patients with Hypertension and Diabetes in Northern Thailand: A Randomized Controlled Trial (ESCAPE Study). Substance abuse Treat Prev Pol (2019). 14(1):10. doi:10.1186/s13011-019-0197-2

54. Latina, J, Fernandez-Jimenez, R, Bansilal, S, Sartori, S, Vedanthan, R, and Lewis, M. Grenada Heart Project-Community Health ActioN to EncouraGe Healthy BEhaviors (GHP-CHANGE): A Randomized Control Peer GroupBased Lifestyle Intervention. Am Heart J (2020). 220:20-8. doi:10.1016/j.ahj. 2019.08.022

55. Anthony, D, Dyson, PA, Lv, J, Thankappan, KR, Fernández, MT, and Matthews, DR. Reducing Health Risk Factors in Workplaces of Low and Middle-Income Countries. Public Health Nurs (2015). 32(5):478-87. doi:10. $1111 / \mathrm{phn} .12189$

56. Neupane, D, McLachlan, CS, Mishra, SR, Olsen, MH, Perry, HB, and Karki, A. Effectiveness of a Lifestyle Intervention Led by Female Community Health Volunteers versus Usual Care in Blood Pressure Reduction (COBIN): an Open-Label, Cluster-Randomised Trial. Lancet Glob Health (2018). 6(1): e66-e73. doi:10.1016/s2214-109x(17)30411-4

57. McGuinness, LA, and Higgins, JPT. Risk-of-bias VISualization (Robvis): An R Package and Shiny Web App for Visualizing Risk-Of-Bias Assessments. Res Synth Methods (2020). 12(1):55-61. doi:10.1002/jrsm.1411

58. McKenzie, JF, Neiger, BL, and Thackeray, R. Planning, Implementing, and Evaluating Health Promotion Programs: A Primer. 6th. San Francisco, CA: Benjamin-Cummings (2012).

59. Pennant, M, Davenport, C, Bayliss, S, Greenheld, W, Marshall, T, and Hyde, C. Community Programs for the Prevention of Cardiovascular Disease: a Systematic Review. Am J Epidemiol (2010). 172(5):501-16. doi:10.1093/aje/kwq171

60. de-Graft Aikins, A, Unwin, N, Agyemang, C, Allotey, P, Campbell, C, and Arhinful, D. Tackling Africa's Chronic Disease burden: from the Local to the Global. Glob Health (2010). 6(1):5. doi:10.1186/1744-8603-6-5
61. Beran, D, and Yudkin, JS. Diabetes Care in Sub-saharan Africa. The Lancet (2006). 368(9548):1689-95. doi:10.1016/s0140-6736(06)69704-3

62. Siddharthan, T, Ramaiya, K, Yonga, G, Mutungi, GN, Rabin, TL, and List, JM. Noncommunicable Diseases in East Africa: Assessing the Gaps in Care and Identifying Opportunities for Improvement. Health Aff (2015). 34(9):1506-13. doi:10.1377/hlthaff.2015.0382

63. Ndejjo, R, Nuwaha, F, Bastiaens, H, Wanyenze, RK, and Musinguzi, G. Cardiovascular Disease Prevention Knowledge and Associated Factors Among Adults in Mukono and Buikwe Districts in Uganda. BMC Public Health (2020). 20(1):1151. doi:10.1186/s12889-020-09264-6

64. Boateng, D, Wekesah, F, Browne, JL, Agyemang, C, Agyei-Baffour, P, and Aikins, Ad.-G Knowledge and Awareness of and Perception towards Cardiovascular Disease Risk in Sub-saharan Africa: A Systematic Review. PloS one. (2017). 12(12):e0189264doi:10.1371/journal.pone.0189264

65. Rothman, AJ. Toward a Theory-Based Analysis of Behavioral Maintenance. Health Psychol (2000). 19(1S):64-9. doi:10.1037/0278-6133.19.suppl1.64

66. Toobert, D, Strycker, LA, Barrera, M, and Glasgow, RE. Seven-year Follow-Up of a Multiple-Health-Behavior Diabetes Intervention. ajhb (2010). 34(6): 680-94. doi:10.5993/ajhb.34.6.5

67. Shroufi, A, Chowdhury, R, Anchala, R, Stevens, S, Blanco, P, and Han, T. Cost Effective Interventions for the Prevention of Cardiovascular Disease in Low and Middle Income Countries: a Systematic Review. BMC public health (2013). 13(1):285. doi:10.1186/1471-2458-13-285

68. Patil, SJ, Ruppar, T, Koopman, RJ, Lindbloom, EJ, Elliott, SG, and Mehr, DR. Effect of Peer Support Interventions on Cardiovascular Disease Risk Factors in Adults with Diabetes: a Systematic Review and Meta-Analysis. BMC public health (2018). 18(1):398. doi:10.1186/s12889-018-5326-8

69. Zhang, X, Devlin, HM, Smith, B, Imperatore, G, Thomas, W, and Lobelo, F. Effect of Lifestyle Interventions on Cardiovascular Risk Factors Among Adults without Impaired Glucose Tolerance or Diabetes: A Systematic Review and Meta-Analysis. PloS one (2017). 12(5):e0176436. doi:10.1371/journal.pone. 0176436

70. Shirinzadeh, M, Afshin-Pour, B, Angeles, R, Gaber, J, and Agarwal, G. The Effect of Community-Based Programs on Diabetes Prevention in Low-And Middle-Income Countries: a Systematic Review and Meta-Analysis. Globalization and health (2019). 15(1):1-13. doi:10.1186/s12992-0190451-4

Copyright (C) 2021 Ndejjo, Hassen, Wanyenze, Musoke, Nuwaha, Abrams, Bastiaens and Musinguzi. This is an open-access article distributed under the terms of the Creative Commons Attribution License (CC BY). The use, distribution or reproduction in other forums is permitted, provided the original author(s) and the copyright owner(s) are credited and that the original publication in this journal is cited, in accordance with accepted academic practice. No use, distribution or reproduction is permitted which does not comply with these terms.

PHR is edited by the Swiss School of Public Health (SSPH+) in a partnership with the Association of Schools of Public Health of the European Region (ASPHER)+ 